\title{
A FREDHOLM ALTERNATIVE FOR QUASILINEAR ELLIPTIC EQUATIONS WITH RIGHT HAND SIDE MEASURE
}

\author{
MICHELE COLTURATO AND MARCO DEGIOVANNI
}

\begin{abstract}
We consider a quasilinear elliptic equation, with right hand side measure, which does not satisfy the usual coercivity assumption. We prove an existence result in the line of the Fredholm alternative. For this purpose we develop a variant of degree theory suited to this setting.
\end{abstract}

\section{INTRODUCTION}

Let $\Omega$ be a bounded and open subset of $\mathbb{R}^{N}$ and let

$$
a: \Omega \times \mathbb{R}^{N} \rightarrow \mathbb{R}^{N}, \quad b: \Omega \times\left(\mathbb{R} \times \mathbb{R}^{N}\right) \rightarrow \mathbb{R}
$$

be two Carathéodory functions. We are interested in the existence of solutions $u$ to the problem

$$
\begin{cases}-\operatorname{div}[a(x, \nabla u)]+b(x, u, \nabla u)=\mu & \text { in } \Omega, \\ u=0 & \text { on } \partial \Omega,\end{cases}
$$

when $\mu$ is a Radon measure on $\Omega$ with bounded total variation.

If $b=0$ and $\{u \mapsto-\operatorname{div}[a(x, \nabla u)]\}$ is a coercive Leray-Lions operator from $W_{0}^{1, p}(\Omega)$ into $W^{-1, p^{\prime}}(\Omega)$, then the problem has been the object of several papers. Let us mention in particular [7], where the existence and uniqueness of an entropy solution is proved when $\mu$ is absolutely continuous with respect to the $p$-capacity, and [12], where the existence and stability of renormalized solutions is proved for a general $\mu$. When $\mu$ is absolutely continuous with respect to the $p$-capacity, the two concepts of entropy solution and renormalized solution agree. A sharper result is proved, in the case $p=N$, in [18], while the case of $\mu$ absolutely continuous with respect to the $p$-capacity is treated also in [19], provided that $p>2-1 / N$.

If $p=2$ and the principal part is linear, then much more is known, both in coercive and noncoercive situations (see e.g. [2, 9, 13, 15, 16, 17, 21, 23, 26]).

On the other hand, if $\mu \in W^{-1, p^{\prime}}(\Omega)$ and the operator

$$
\{u \mapsto-\operatorname{div}[a(x, \nabla u)]+b(x, u, \nabla u)\}
$$

is well defined and continuous from $W_{0}^{1, p}(\Omega)$ into $W^{-1, p^{\prime}}(\Omega)$, then the problem has been treated, under various assumptions, also for $p \neq 2$ in the noncoercive case. Let us mention, in particular, the results in the line of the Fredholm alternative proved in [4] and then developed with detailed descriptions, when resonance occurs at the principal eigenvalue (see [25] and references therein).

2010 Mathematics Subject Classification. 35J66, 35R06, 47H11.

Key words and phrases. Quasilinear elliptic equations, right hand side measure, degree theory.

The second author is member of the Gruppo Nazionale per l'Analisi Matematica, la Probabilità e le loro Applicazioni (GNAMPA) of the Istituto Nazionale di Alta Matematica (INdAM). 
We are interested in a result in the same direction, when $\mu$ is a Radon measure which has bounded total variation and is absolutely continuous with respect to the p-capacity. More precisely, we assume that:

(i) there exist $1<p<\infty, \alpha_{0}, \alpha_{2} \in L^{1}(\Omega), \alpha_{1} \in L^{p^{\prime}}(\Omega), \beta \in \mathbb{R}$ and $\nu>0$ such that

$$
\begin{aligned}
& a(x, \xi) \cdot \xi \geq \nu|\xi|^{p}-\alpha_{0}(x), \\
& |a(x, \xi)| \leq \alpha_{1}(x)+\beta|\xi|^{p-1}, \\
& |b(x, s, \xi)| \leq \alpha_{2}(x)+\beta|s|^{p-1}+\beta|\xi|^{p-1}
\end{aligned}
$$

for a.e. $x \in \Omega$ and every $s \in \mathbb{R}, \xi \in \mathbb{R}^{N}$; such a $p$ is clearly unique;

(ii) we have

$$
[a(x, \xi)-a(x, \eta)] \cdot(\xi-\eta)>0
$$

for a.e. $x \in \Omega$ and every $\xi, \eta \in \mathbb{R}^{N}$ with $\xi \neq \eta$;

(iii) there exist two Carathéodory functions

$$
a_{\infty}: \Omega \times \mathbb{R}^{N} \rightarrow \mathbb{R}^{N}, \quad b_{\infty}: \Omega \times\left(\mathbb{R} \times \mathbb{R}^{N}\right) \rightarrow \mathbb{R},
$$

such that

$$
\left[a_{\infty}(x, \xi)-a_{\infty}(x, \eta)\right] \cdot(\xi-\eta)>0,
$$

for a.e. $x \in \Omega$ and every $\xi, \eta \in \mathbb{R}^{N}$ with $\xi \neq \eta$, and such that

$$
\lim _{k} \frac{a\left(x, \tau_{k} \xi_{k}\right)}{\tau_{k}^{p-1}}=a_{\infty}(x, \xi), \quad \lim _{k} \frac{b\left(x, \tau_{k} s_{k}, \tau_{k} \xi_{k}\right)}{\tau_{k}^{p-1}}=b_{\infty}(x, s, \xi),
$$

for a.e. $x \in \Omega$, whenever $\tau_{k} \rightarrow+\infty, s_{k} \rightarrow s$ and $\xi_{k} \rightarrow \xi$.

It easily follows that

$$
\begin{aligned}
& a_{\infty}(x, \xi) \cdot \xi \geq \nu|\xi|^{p} \\
& \left|a_{\infty}(x, \xi)\right| \leq \beta|\xi|^{p-1} \\
& \left|b_{\infty}(x, s, \xi)\right| \leq \beta|s|^{p-1}+\beta|\xi|^{p-1} \\
& a_{\infty}(x, \tau \xi)=\tau^{p-1} a_{\infty}(x, \xi) \\
& b_{\infty}(x, \tau s, \tau \xi)=\tau^{p-1} b_{\infty}(x, s, \xi)
\end{aligned}
$$

for a.e. $x \in \Omega$ and every $\tau, s \in \mathbb{R}, \xi \in \mathbb{R}^{N}$ with $\tau \geq 0$.

We aim to prove the next result.

Theorem 1.1. Under hypotheses $(i)-(i i i)$, assume also that

$$
a_{\infty}(x,-\xi)=-a_{\infty}(x, \xi), \quad b_{\infty}(x,-s,-\xi)=-b_{\infty}(x, s, \xi),
$$

for a.e. $x \in \Omega$ and every $s \in \mathbb{R}$ and $\xi \in \mathbb{R}^{N}$.

Then one at least of the following assertions is true:

(a) the problem

$$
\left\{\begin{array}{l}
u \in W_{0}^{1, p}(\Omega) \backslash\{0\} \\
-\operatorname{div}\left[a_{\infty}(x, \nabla u)\right]+b_{\infty}(x, u, \nabla u)=0 \quad \text { in } W^{-1, p^{\prime}}(\Omega)
\end{array}\right.
$$

admits a solution; 
(b) for every Radon measure $\mu$, which has bounded total variation and is absolutely continuous with respect to the p-capacity, problem (1.1) admits an entropy solution $u$ (see the next Section 3 for the definition of entropy solution in this setting).

In order to prove Theorem 1.1, we first develop an adaptation of degree theory to our setting. Since the problems with right hand side measure are usually treated by an approximation procedure involving operators of Leray-Lions type, we choose a development of the degree for maps of class $(S)_{+}$(see $\left.[10,22,24]\right)$, which naturally acts in the same setting.

In Section 2 we recall the main facts concerning entropy solutions to problems of the form

$$
\begin{cases}-\operatorname{div}[a(x, \nabla u)]=\mu & \text { in } \Omega, \\ u=0 & \text { on } \partial \Omega,\end{cases}
$$

and prove some auxiliary results. Then we introduce, in Section 3, the adaptation of the topological degree and state its properties. With this tool at hand, in Section 4 we prove Theorem 1.1. The subsequent sections are devoted to the construction of such a degree and the proof of its properties.

Since each Radon measure with bounded total variation belongs to $W^{-1, p^{\prime}}$ if $p>N$, we have no novelty in this case. Therefore, from now on we only consider the case $1<p \leq N$.

In the following, we denote by \|\|$_{p}$ the usual $L^{p}$-norm and by \|\|$_{-1, p^{\prime}}$ the norm in $W^{-1, p^{\prime}}(\Omega)$ dual to the norm $\|\nabla u\|_{p}$ in $W_{0}^{1, p}(\Omega)$. If $s \in \mathbb{R}$, we set $s^{ \pm}=\max \{ \pm s, 0\}$.

\section{Entropy SOLUtions}

From now on, $\Omega$ will denote a bounded and open subset of $\mathbb{R}^{N}$ with $N \geq 2$. According to [7], if $1<p \leq N$ we denote by $\mathcal{M}_{b}^{p}(\Omega)$ the set of Radon measures $\mu$ on $\Omega$ whose total variation $|\mu|$ is bounded and absolutely continuous with respect to the $p$-capacity.

According to [3, 7], we also denote by $\mathcal{T}_{0}^{1, p}(\Omega)$ the set of (classes of equivalence of) functions $u: \Omega \rightarrow[-\infty,+\infty]$ such that $|u|<+\infty$ a.e. in $\Omega$ and $T_{k}(u) \in W_{0}^{1, p}(\Omega)$ for any $k>0$, where

$$
T_{k}(s)= \begin{cases}s & \text { if }|s| \leq k, \\ k \frac{s}{|s|} & \text { if }|s|>k .\end{cases}
$$

If $u \in \mathcal{T}_{0}^{1, p}(\Omega)$, there exists one and only one measurable (class of equivalence) $\nabla u: \Omega \rightarrow \mathbb{R}^{N}$ such that $g(u) \in W_{0}^{1, p}(\Omega)$ and $\nabla[g(u)]=g^{\prime}(u) \nabla u$ a.e. in $\Omega$, whenever $g: \mathbb{R} \rightarrow \mathbb{R}$ is Lipschitz continuous with $g(0)=0$ and $g^{\prime}(s)=0$ outside some compact subset of $\mathbb{R}$.

According to [12], any $u \in \mathcal{T}_{0}^{1, p}(\Omega)$ has a Borel and capp-quasi continuous representative $\tilde{u}: \Omega \rightarrow[-\infty,+\infty]$, defined up to a set of null $p$-capacity, which we still denote by $u$. Of course, the set $\{|u|=+\infty\}$ has null measure, but could have positive $p$-capacity.

If $u, u_{1}, u_{2} \in \mathcal{T}_{0}^{1, p}(\Omega)$ and $t \in \mathbb{R}$, it is easily seen that

$$
\text { tu, } \max \left\{u_{1}, u_{2}\right\}, \quad \min \left\{u_{1}, u_{2}\right\} \in \mathcal{T}_{0}^{1, p}(\Omega)
$$


with

$$
\begin{aligned}
& \nabla(t u)=t \nabla u, \\
& \nabla u_{1}=\nabla u_{2}, \text { a.e. in }\left\{u_{1}=u_{2}\right\}, \\
& \nabla \max \left\{u_{1}, u_{2}\right\}=\chi_{\left\{u_{1}>u_{2}\right\}} \nabla u_{1}+\chi_{\left\{u_{2} \geq u_{1}\right\}} \nabla u_{2}, \\
& \nabla \min \left\{u_{1}, u_{2}\right\}=\chi_{\left\{u_{1}<u_{2}\right\}} \nabla u_{1}+\chi_{\left\{u_{2} \leq u_{1}\right\}} \nabla u_{2} .
\end{aligned}
$$

On the contrary, one cannot say (see [3]) that $u_{1}+u_{2} \in \mathcal{T}_{0}^{1, p}(\Omega)$. For reader's convenience, we also provide a proof of the next result.

Proposition 2.1. Let $u_{1}, u_{2} \in \mathcal{T}_{0}^{1, p}(\Omega)$ with $\nabla u_{1}=\nabla u_{2}$ a.e. in $\Omega$. Assume also that

$$
\liminf _{k \rightarrow+\infty} \frac{1}{k^{p}} \int_{\left\{k<\left|u_{1}\right|<2 k\right\}}\left|\nabla u_{1}\right|^{p} d x<+\infty .
$$

Then $u_{1}=u_{2}$ a.e. in $\Omega$.

Proof. Let $\vartheta: \mathbb{R} \rightarrow[0,1]$ be a smooth function with $\vartheta(s)=0$ for $|s| \leq 1$ and $\vartheta(s)=1$ for $|s| \geq 2$. Then we have

$$
T_{2 k}\left(u_{1}\right), T_{1}\left(u_{2}-T_{2 k}\left(u_{1}\right)\right), \vartheta\left(u_{1} / k\right) \in W_{0}^{1, p}(\Omega) \cap L^{\infty}(\Omega),
$$

whence

$$
\begin{aligned}
T_{1}\left(u_{2}-u_{1}\right)\left(1-\vartheta\left(u_{1} / k\right)\right) & \\
= & T_{1}\left(u_{2}-T_{2 k}\left(u_{1}\right)\right)\left(1-\vartheta\left(u_{1} / k\right)\right) \in W_{0}^{1, p}(\Omega) \cap L^{\infty}(\Omega)
\end{aligned}
$$

with

$$
\left|\nabla\left[T_{1}\left(u_{2}-u_{1}\right)\left(1-\vartheta\left(u_{1} / k\right)\right)\right]\right| \leq \frac{\left\|\vartheta^{\prime}\right\|_{\infty}}{k} \chi_{\left\{k<\left|u_{1}\right|<2 k\right\}}\left|\nabla u_{1}\right| .
$$

If $\left(k_{j}\right)$ is a sequence with $k_{j} \rightarrow+\infty$ and

$$
\sup _{j \in \mathbb{N}} \frac{1}{k_{j}^{p}} \int_{\left\{k_{j}<\left|u_{1}\right|<2 k_{j}\right\}}\left|\nabla u_{1}\right|^{p} d x<+\infty,
$$

it follows that $\left(T_{1}\left(u_{2}-u_{1}\right)\left(1-\vartheta\left(u_{1} / k_{j}\right)\right)\right)$ is convergent both to $T_{1}\left(u_{2}-u_{1}\right)$ a.e. in $\Omega$ and to 0 weakly in $W_{0}^{1, p}(\Omega)$. Then $T_{1}\left(u_{2}-u_{1}\right)=0$ a.e. in $\Omega$ and the assertion follows.

Remark 2.2. Let $\Omega=\left\{x \in \mathbb{R}^{N}:|x|<1\right\}$ and let $u_{j}(x)=v_{j}(|x|)$, where

$$
\begin{aligned}
& v_{1}(r)= \begin{cases}\frac{1-r}{(1-2 r)^{2} r} & \text { if } 0<r<\frac{1}{2} \text { or } \frac{1}{2}<r<1, \\
+\infty & \text { if } r=0 \text { or } r=\frac{1}{2} .\end{cases} \\
& v_{2}(r)= \begin{cases}1+\frac{1-r}{(1-2 r)^{2} r} & \text { if } 0<r<\frac{1}{2}, \\
\frac{1-r}{(1-2 r)^{2} r} & \text { if } \frac{1}{2}<r<1, \\
+\infty & \text { if } r=0 \text { or } r=\frac{1}{2} .\end{cases}
\end{aligned}
$$

Then $u_{1}, u_{2} \in \mathcal{T}_{0}^{1, p}(\Omega)$ with $\nabla u_{1}=\nabla u_{2}$ a.e. in $\Omega$, but it is false that $u_{1}=u_{2}$ a.e. in $\Omega$. One can also observe that $-u_{1} \in \mathcal{T}_{0}^{1, p}(\Omega)$, but $u_{2}+\left(-u_{1}\right) \notin \mathcal{T}_{0}^{1, p}(\Omega)$. 
We are also interested in a smaller space, suggested by the techniques of $[5,6,23]$. Let us denote by $\varphi_{p}: \mathbb{R} \rightarrow \mathbb{R}$ the increasing $C^{\infty}$-diffeomorphism such that

$$
\varphi_{p}^{\prime}(s)=\frac{1}{\left\{\left(1+s^{2}\right)\left[\log \left(e+s^{2}\right)\right]^{4}\right\}^{\frac{1}{2 p}}}, \quad \varphi_{p}(0)=0 .
$$

Then we denote by $\Phi_{0}^{1, p}(\Omega)$ the set of (classes of equivalence of) functions $u: \Omega \rightarrow \mathbb{R}$ such that $\varphi_{p}(u) \in W_{0}^{1, p}(\Omega)$. It is easily seen that

$$
W_{0}^{1, p}(\Omega) \subseteq \Phi_{0}^{1, p}(\Omega) \subseteq \mathcal{T}_{0}^{1, p}(\Omega)
$$

and that $\nabla\left[\varphi_{p}(u)\right]=\varphi_{p}^{\prime}(u) \nabla u$ a.e. in $\Omega$, where $\nabla u$ has to be understood in the sense of $\mathcal{T}_{0}^{1, p}(\Omega)$. Moreover, any $u \in \Phi_{0}^{1, p}(\Omega)$ has a Borel and cap $p_{\text {-quasi continuous }}$ representative $\tilde{u}: \Omega \rightarrow \mathbb{R}$, defined up to a set of null $p$-capacity, which we still denote by $u$.

Since $\left\{u \mapsto \varphi_{p}(u)\right\}$ is bijective from $\Phi_{0}^{1, p}(\Omega)$ onto $W_{0}^{1, p}(\Omega)$, there is a natural structure of complete metric space on $\Phi_{0}^{1, p}(\Omega)$ which makes $\left\{u \mapsto \varphi_{p}(u)\right\}$ an isometry. In particular, the distance function is given by

$$
d(u, v)=\left\|\nabla\left[\varphi_{p}(u)\right]-\nabla\left[\varphi_{p}(v)\right]\right\|_{p} \quad \text { for any } u, v \in \Phi_{0}^{1, p}(\Omega) .
$$

Proposition 2.3. Let $u_{1} \in \Phi_{0}^{1, p}(\Omega)$ and $u_{2} \in \mathcal{T}_{0}^{1, p}(\Omega)$ with $\nabla u_{1}=\nabla u_{2}$ a.e. in $\Omega$. Then $u_{1}=u_{2}$ a.e. in $\Omega$.

Proof. Taking into account the behavior of $\varphi_{p}^{\prime}$ at infinity, from

$$
\int_{\Omega}\left|\varphi_{p}^{\prime}\left(u_{1}\right)\right|^{p}\left|\nabla u_{1}\right|^{p} d x<+\infty
$$

we infer that

$$
\int_{\Omega} \frac{\left|\nabla u_{1}\right|^{p}}{1+\left|u_{1}\right|^{p}} d x<+\infty
$$

Since

$$
\frac{1}{k^{p}} \int_{\left\{k<\left|u_{1}\right|<2 k\right\}}\left|\nabla u_{1}\right|^{p} d x \leq 2^{p+1} \int_{\left\{k<\left|u_{1}\right|<2 k\right\}} \frac{\left|\nabla u_{1}\right|^{p}}{1+\left|u_{1}\right|^{p}} d x
$$

for every $k \geq 1$, by Proposition 2.1 the assertion follows.

Now let $a: \Omega \times \mathbb{R}^{N} \rightarrow \mathbb{R}^{N}$ be a Carathéodory function such that:

$\left(a_{1}\right)$ there exist $1<p \leq N, \alpha_{0} \in L^{1}(\Omega), \alpha_{1} \in L^{p^{\prime}}(\Omega), \beta_{1} \in \mathbb{R}$ and $\nu>0$ such that

$$
\begin{aligned}
& a(x, \xi) \cdot \xi \geq \nu|\xi|^{p}-\alpha_{0}(x), \\
& |a(x, \xi)| \leq \alpha_{1}(x)+\beta_{1}|\xi|^{p-1},
\end{aligned}
$$

for a.e. $x \in \Omega$ and every $\xi \in \mathbb{R}^{N}$;

$\left(a_{2}\right)$ we have

$$
[a(x, \xi)-a(x, \eta)] \cdot(\xi-\eta)>0
$$

for a.e. $x \in \Omega$ and every $\xi, \eta \in \mathbb{R}^{N}$ with $\xi \neq \eta$.

Definition 2.4. Given $\mu \in \mathcal{M}_{b}^{p}(\Omega)$, we say that $u$ is an entropy solution of

$$
\begin{cases}-\operatorname{div}[a(x, \nabla u)]=\mu & \text { in } \Omega, \\ u=0 & \text { on } \partial \Omega,\end{cases}
$$


if $u \in \mathcal{T}_{0}^{1, p}(\Omega)$ and

$$
\int_{\Omega} a(x, \nabla u) \cdot \nabla\left[T_{k}(u-v)\right] d x \leq \int_{\Omega} T_{k}(u-v) d \mu \quad \forall k>0, \forall v \in C_{c}^{\infty}(\Omega) .
$$

If $u$ is an entropy solution of (2.1), then $u$ actually satisfies the equality and for a much larger class of test functions. Following the original idea of [8], we aim to prove a result in this direction.

If $h, k \geq 0$, let $T_{h, k}: \mathbb{R} \rightarrow \mathbb{R}$ be the odd function such that

$$
T_{h, k}(s)= \begin{cases}0 & \text { if } 0 \leq s \leq h, \\ s-h & \text { if } h<s<h+k, \\ k & \text { if } s \geq h+k .\end{cases}
$$

Then denote by $\widetilde{\mathcal{T}}_{0}^{1, p}(\Omega)$ the set of (classes of equivalence of) functions $u: \Omega \rightarrow$ $[-\infty,+\infty]$ such that $|u|<+\infty$ a.e. in $\Omega$ and $T_{\varepsilon, k}(u) \in W_{0}^{1, p}(\Omega)$ whenever $\varepsilon>0$ and $k>0$. It is easily seen that $\mathcal{T}_{0}^{1, p}(\Omega) \subseteq \widetilde{\mathcal{T}}_{0}^{1, p}(\Omega)$. Moreover, if $u \in \widetilde{\mathcal{T}}_{0}^{1, p}(\Omega)$, there exists one and only one measurable (class of equivalence) $\nabla u: \Omega \rightarrow \mathbb{R}^{N}$ such that $\nabla u=0$ a.e. on $\{u=0\}$ and such that $g(u) \in W_{0}^{1, p}(\Omega)$ with $\nabla[g(u)]=g^{\prime}(u) \nabla u$ a.e. in $\Omega$, whenever $g: \mathbb{R} \rightarrow \mathbb{R}$ is Lipschitz continuous with $g(0)=0$ and $g^{\prime}(s)=0$ outside some compact subset of $]-\infty, 0[\cup] 0,+\infty\left[\right.$. If $u \in \mathcal{T}_{0}^{1, p}(\Omega)$, then the gradient of $u$ in the sense of $\mathcal{T}_{0}^{1, p}(\Omega)$ agrees with that in the sense of $\widetilde{\mathcal{T}}_{0}^{1, p}(\Omega)$.

As in the case of $\mathcal{T}_{0}^{1, p}(\Omega)$, any $u \in \widetilde{\mathcal{T}}_{0}^{1, p}(\Omega)$ has a Borel and cap ${ }^{\text {-quasi continuous }}$ representative $\tilde{u}: \Omega \rightarrow[-\infty,+\infty]$, defined up to a set of null $p$-capacity, which we still denote by $u$. Finally, let us point out that, if $u \in \widetilde{\mathcal{T}}_{0}^{1, p}(\Omega)$, we have $|u|^{t-1} u \in \widetilde{\mathcal{T}}_{0}^{1, p}(\Omega)$ whenever $t>0$.

Theorem 2.5. Let $\mu \in \mathcal{M}_{b}^{p}(\Omega)$ and let $u$ be an entropy solution of (2.1). Then we have

$$
\begin{aligned}
\int_{\Omega}(a(x, \nabla u) & \cdot \nabla v)^{+} d x+\int_{\Omega}(v \gamma)^{-} d|\mu| \\
& =\int_{\Omega}(a(x, \nabla u) \cdot \nabla v)^{-} d x+\int_{\Omega}(v \gamma)^{+} d|\mu| \quad \forall v \in \widetilde{\mathcal{T}}_{0}^{1, p}(\Omega),
\end{aligned}
$$

where $d \mu=\gamma d|\mu|$ and $\gamma$ is a Borel function with $|\gamma|=1|\mu|$-a.e. in $\Omega$.

Proof. As in [3, Lemma 3.3], we have

$$
\begin{aligned}
& \int_{\Omega} a(x, \nabla u) \cdot \nabla\left[T_{k}(u-v)\right] d x \leq \int_{\Omega} T_{k}(u-v) \gamma d|\mu| \\
& \forall k>0, \forall v \in W_{0}^{1, p}(\Omega) \cap L^{\infty}(\Omega) .
\end{aligned}
$$

Now assume that $v \in W_{0}^{1, p}(\Omega) \cap L^{\infty}(\Omega)$ with $(a(x, \nabla u) \cdot \nabla v)^{+} \in L^{1}(\Omega)$ and take $k>\|v\|_{\infty}$. By (2.3) for every $t>0$ we have

$$
\int_{\Omega} a(x, \nabla u) \cdot \nabla\left[T_{t k}(u-t v)\right] d x \leq \int_{\Omega} T_{t k}(u-t v) \gamma d|\mu| .
$$

Since $T_{t k}(t s)=t T_{k}(s)$, it follows

$$
\int_{\Omega} a(x, \nabla u) \cdot \nabla\left[T_{k}\left(\frac{u}{t}-v\right)\right] d x \leq \int_{\Omega} T_{k}\left(\frac{u}{t}-v\right) \gamma d|\mu|,
$$


whence

$$
\begin{aligned}
\int_{\Omega} T_{k}\left(\frac{u}{t}-v\right) \gamma d|\mu| & \geq \frac{1}{t} \int_{\left\{\left|\frac{u}{t}-v\right|<k\right\}} a(x, \nabla u) \cdot \nabla u d x \\
& -\int_{\left\{\left|\frac{u}{t}-v\right|<k\right\}} a(x, \nabla u) \cdot \nabla v d x \\
& \geq-\frac{1}{t} \int_{\Omega} \alpha_{0} d x-\int_{\left\{\left|\frac{u}{t}-v\right|<k\right\}} a(x, \nabla u) \cdot \nabla v d x .
\end{aligned}
$$

Since $(a(x, \nabla u) \cdot \nabla v)^{+} \in L^{1}(\Omega)$, passing to the lower limit as $t \rightarrow+\infty$ and applying Fatou's lemma at the right hand side, we get

$$
\int_{\Omega} a(x, \nabla u) \cdot \nabla v d x \geq \int_{\Omega} v \gamma d|\mu| .
$$

It follows $a(x, \nabla u) \cdot \nabla v \in L^{1}(\Omega)$, which allows to apply the same argument also to $-v$, obtaining

$$
\int_{\Omega} a(x, \nabla u) \cdot \nabla v d x=\int_{\Omega} v \gamma d|\mu|
$$

Consider now $v \in \widetilde{\mathcal{T}}_{0}^{1, p}(\Omega)$ with $(a(x, \nabla u) \cdot \nabla v)^{+} \in L^{1}(\Omega)$ and $(v \gamma)^{-} \in L^{1}(\Omega,|\mu|)$. Then $T_{1 / k, k}(v) \in W_{0}^{1, p}(\Omega) \cap L^{\infty}(\Omega)$ with

$$
\left(a(x, \nabla u) \cdot \nabla T_{1 / k, k}(v)\right)^{+} \leq(a(x, \nabla u) \cdot \nabla v)^{+}, \quad\left(T_{1 / k, k}(v) \gamma\right)^{-} \leq(v \gamma)^{-} .
$$

First of all, it follows $a(x, \nabla u) \cdot \nabla T_{1 / k, k}(v) \in L^{1}(\Omega)$ and

$$
\int_{\Omega} a(x, \nabla u) \cdot \nabla T_{1 / k, k}(v) d x=\int_{\Omega} T_{1 / k, k}(v) \gamma d|\mu|
$$

Then, if $k \rightarrow \infty$, from Fatou's lemma we infer that

$$
\int_{\Omega} a(x, \nabla u) \cdot \nabla v d x \geq \int_{\Omega} v \gamma d|\mu|
$$

It follows $a(x, \nabla u) \cdot \nabla v \in L^{1}(\Omega)$ and $v \gamma \in L^{1}(\Omega,|\mu|)$, so that we can argue on $-v$, obtaining

$$
\int_{\Omega} a(x, \nabla u) \cdot \nabla v d x=\int_{\Omega} v \gamma d|\mu|
$$

If $v \in \tilde{\mathcal{T}}_{0}^{1, p}(\Omega)$ with $(a(x, \nabla u) \cdot \nabla v)^{-} \in L^{1}(\Omega)$ and $(v \gamma)^{+} \in L^{1}(\Omega,|\mu|)$, the argument is analogous. Otherwise, both sides of $(2.2)$ are $+\infty$.

In the construction of the degree, a key role will be played by the next regularity result.

Theorem 2.6. If $\mu \in \mathcal{M}_{b}^{p}(\Omega)$ and $u$ is an entropy solution of (2.1), then $u \in$ $\Phi_{0}^{1, p}(\Omega)$. In particular, the set $\{|u|=+\infty\}$ has null p-capacity. Moreover, if we define an increasing and bounded $C^{\infty}$-function $\psi: \mathbb{R} \rightarrow \mathbb{R}$ by

$$
\psi^{\prime}(s)=\frac{1}{\left\{\left(1+s^{2}\right)\left[\log \left(e+s^{2}\right)\right]^{4}\right\}^{\frac{1}{2}}}=\left(\varphi_{p}^{\prime}(s)\right)^{p}, \quad \psi(0)=0,
$$


then $\psi(u) \in W_{0}^{1, p}(\Omega) \cap L^{\infty}(\Omega), \psi^{\prime}(u) a(x, \nabla u) \cdot \nabla u \in L^{1}(\Omega)$ and

$$
\begin{gathered}
\int_{\Omega} \psi^{\prime}(u) a(x, \nabla u) \cdot \nabla u d x=\int_{\Omega} \psi(u) d \mu, \\
\nu \int_{\Omega}\left|\nabla\left[\varphi_{p}(u)\right]\right|^{p} d x \leq\|\psi\|_{\infty}|\mu|(\Omega)+\left\|\alpha_{0}\right\|_{1} .
\end{gathered}
$$

Proof. By Theorem 2.5 we have

whence

$$
\int_{\Omega} a(x, \nabla u) \cdot \nabla\left[\psi\left(T_{k}(u)\right)\right] d x=\int_{\Omega} \psi\left(T_{k}(u)\right) d \mu,
$$

$$
\begin{aligned}
\nu \int_{\Omega}\left|\nabla\left[\varphi_{p}\left(T_{k}(u)\right)\right]\right|^{p} d x & =\nu \int_{\{|u|<k\}} \psi^{\prime}(u)|\nabla u|^{p} d x \\
& \leq \int_{\{|u|<k\}} \psi^{\prime}(u)\left(a(x, \nabla u) \cdot \nabla u+\alpha_{0}\right) d x \\
& =\int_{\Omega} \psi\left(T_{k}(u)\right) d \mu+\int_{\{|u|<k\}} \psi^{\prime}(u) \alpha_{0} d x
\end{aligned}
$$

Since $\psi^{\prime}(s) \leq 1$, it follows

$$
\nu \int_{\Omega}\left|\nabla \varphi_{p}\left(T_{k}(u)\right)\right|^{p} d x \leq\|\psi\|_{\infty}|\mu|(\Omega)+\left\|\alpha_{0}\right\|_{1}
$$

so that $\left(\varphi_{p}\left(T_{k}(u)\right)\right)$ is bounded in $W_{0}^{1, p}(\Omega)$. Therefore $\varphi_{p}(u) \in W_{0}^{1, p}(\Omega)$ with

$$
\nu \int_{\Omega}\left|\nabla\left[\varphi_{p}(u)\right]\right|^{p} d x \leq\|\psi\|_{\infty}|\mu|(\Omega)+\left\|\alpha_{0}\right\|_{1} .
$$

Since $\psi^{\prime}(s) \leq \varphi_{p}^{\prime}(s)$, a fortiori we have that $\left(\psi\left(T_{k}(u)\right)\right)$ is bounded in $W_{0}^{1, p}(\Omega)$, so that $\psi(u) \in W_{0}^{1, p}(\Omega) \cap L^{\infty}(\Omega)$. Coming back to the equality

$$
\begin{aligned}
& \int_{\{|u|<k\}} \psi^{\prime}(u)\left(a(x, \nabla u) \cdot \nabla u+\alpha_{0}\right) d x \\
&=\int_{\Omega} \psi\left(T_{k}(u)\right) d \mu+\int_{\{|u|<k\}} \psi^{\prime}(u) \alpha_{0} d x,
\end{aligned}
$$

from the monotone convergence theorem we infer that $\psi^{\prime}(u) a(x, \nabla u) \cdot \nabla u \in L^{1}(\Omega)$ and

$$
\int_{\Omega} \psi^{\prime}(u) a(x, \nabla u) \cdot \nabla u d x=\int_{\Omega} \psi(u) d \mu .
$$

Remark 2.7. By Theorem 2.6, in the definition of entropy solution it is equivalent to require $u \in \mathcal{T}_{0}^{1, p}(\Omega)$ or $u \in \Phi_{0}^{1, p}(\Omega)$.

Now let us recall the main result on entropy solutions.

Theorem 2.8. For every $\mu \in \mathcal{M}_{b}^{p}(\Omega)$, there exists one and only one entropy solution $u$ of (2.1). Moreover, if $\mu_{1}, \mu_{2} \in \mathcal{M}_{b}^{p}(\Omega)$ satisfy

$$
\int_{\Omega} v d \mu_{1} \leq \int_{\Omega} v d \mu_{2} \quad \text { for any } v \in C_{c}^{\infty}(\Omega) \text { with } v \geq 0
$$

and $u_{1}, u_{2} \in \Phi_{0}^{1, p}(\Omega)$ are the corresponding entropy solutions of (2.1), it follows $u_{1} \leq u_{2}$ a.e. in $\Omega$. 
Proof. If $u$ is an entropy solution of (2.1), we clearly have

$$
\lim _{h} \int_{\{|u| \geq h\}} \alpha_{0} d x=0 .
$$

As in [7, Formula (7)], it follows

$$
\lim _{h} \int_{\{h \leq|u| \leq h+k\}}|\nabla u|^{p} d x=0 \quad \text { for any } k>0 .
$$

Then the existence and uniqueness of the entropy solution $u$ can be proved as in [7] (see also [3] and the proof of the next Lemma 5.4 for the existence part). More specifically, the order preserving can be proved as in [19, Theorem 2.5], where the condition $p>2-1 / N$ is assumed. However, the same argument works in our case. We sketch it for reader's convenience.

By Theorem 2.6 we have $u_{1}, u_{2} \in \Phi_{0}^{1, p}(\Omega)$. Moreover, by Theorem 2.5, it holds whenever $0<k<h$

$$
\begin{aligned}
\int_{\Omega}\left[T_{k}\left(u_{1}-T_{h}\left(u_{2}\right)\right)\right]^{+} d \mu_{1}= & \int_{\Omega} a\left(x, \nabla u_{1}\right) \cdot \nabla\left[T_{k}\left(u_{1}-T_{h}\left(u_{2}\right)\right)\right]^{+} d x \\
\geq & \int_{\left\{\left|u_{1}\right|<h,\left|u_{2}\right|<h, 0<u_{1}-u_{2}<k\right\}} a\left(x, \nabla u_{1}\right) \cdot \nabla\left(u_{1}-u_{2}\right) d x \\
& -\int_{\left\{h \leq\left|u_{1}\right|<h+k, h-k<\left|u_{2}\right|<h\right\}}\left|a\left(x, \nabla u_{1}\right)\right|\left|\nabla u_{2}\right| d x \\
& -\int_{\left\{\left|u_{1}\right| \geq h\right\}} \alpha_{0} d x-\int_{\left\{\left|u_{2}\right| \geq h\right\}} \alpha_{0} d x, \\
\int_{\Omega}\left[T_{k}\left(u_{2}-T_{h}\left(u_{1}\right)\right)\right]^{-} d \mu_{2}= & \int_{\Omega} a\left(x, \nabla u_{2}\right) \cdot \nabla\left[T_{k}\left(u_{2}-T_{h}\left(u_{1}\right)\right)\right]^{-} d x \\
\leq & \int_{\left\{\left|u_{1}\right|<h,\left|u_{2}\right|<h, 0<u_{1}-u_{2}<k\right\}} a\left(x, \nabla u_{2}\right) \cdot \nabla\left(u_{1}-u_{2}\right) d x \\
& +\int_{\left\{h \leq\left|u_{2}\right|<h+k, h-k<\left|u_{1}\right|<h\right\}}\left|a\left(x, \nabla u_{2}\right)\right|\left|\nabla u_{1}\right| d x \\
& +\int_{\left\{\left|u_{2}\right| \geq h\right\}} \alpha_{0} d x+\int_{\left\{\left|u_{1}\right| \geq h\right\}} \alpha_{0} d x .
\end{aligned}
$$

It follows

$$
\begin{gathered}
\int_{\Omega}\left[T_{k}\left(u_{1}-T_{h}\left(u_{2}\right)\right)\right]^{+} d \mu_{1}-\int_{\Omega}\left[T_{k}\left(u_{2}-T_{h}\left(u_{1}\right)\right)\right]^{-} d \mu_{2} \\
\geq \int_{\left\{\left|u_{1}\right|<h,\left|u_{2}\right|<h, 0<u_{1}-u_{2}<k\right\}}\left[a\left(x, \nabla u_{1}\right)-a\left(x, \nabla u_{2}\right)\right] \cdot \nabla\left(u_{1}-u_{2}\right) d x \\
-\int_{\left\{h \leq\left|u_{1}\right|<h+k, h-k<\left|u_{2}\right|<h\right\}}\left|a\left(x, \nabla u_{1}\right)\right|\left|\nabla u_{2}\right| d x \\
-\int_{\left\{h \leq\left|u_{2}\right|<h+k, h-k<\left|u_{1}\right|<h\right\}}\left|a\left(x, \nabla u_{2}\right)\right|\left|\nabla u_{1}\right| d x \\
-2 \int_{\left\{\left|u_{1}\right| \geq h\right\}} \alpha_{0} d x-2 \int_{\left\{\left|u_{2}\right| \geq h\right\}} \alpha_{0} d x .
\end{gathered}
$$


Passing to the limit as $h \rightarrow+\infty$ and taking into account (2.6), we get

$$
\begin{aligned}
0 \geq-\int_{\Omega}\left[T_{k}\left(u_{1}-u_{2}\right)\right]^{+} & d\left(\mu_{2}-\mu_{1}\right) \\
& \geq \int_{\left\{0<u_{1}-u_{2}<k\right\}}\left[a\left(x, \nabla u_{1}\right)-a\left(x, \nabla u_{2}\right)\right] \cdot \nabla\left(u_{1}-u_{2}\right) d x,
\end{aligned}
$$

whence $\nabla u_{1}=\nabla u_{2}$ a.e. in $\left\{u_{1}>u_{2}\right\}$, namely $\nabla\left[\max \left\{u_{1}, u_{2}\right\}\right]=\nabla u_{2}$ a.e. in $\Omega$. Since $\max \left\{u_{1}, u_{2}\right\} \in \mathcal{T}_{0}^{1, p}(\Omega)$ and $u_{2} \in \Phi_{0}^{1, p}(\Omega)$, by Proposition 2.3 we infer that $\max \left\{u_{1}, u_{2}\right\}=u_{2}$, namely $u_{1} \leq u_{2}$.

Proposition 2.9. The following facts hold:

(a) if $u \in \Phi_{0}^{1, p}(\Omega)$, then $|\nabla u|^{p-1} \in L^{q}(\Omega)$ and $|u|^{p-1} \in L^{r}(\Omega)$, whenever $q<$ $\frac{N}{N-1}$ and $r<\frac{N}{N-p}(r<\infty$ if $p=N)$;

(b) if $\left(u_{n}\right)$ is bounded in $\Phi_{0}^{1, p}(\Omega)$, then $\left(\left|\nabla u_{n}\right|^{p-1}\right)$ is bounded in $L^{q}(\Omega)$, whenever $q<\frac{N}{N-1}$; moreover, there exists $u \in \Phi_{0}^{1, p}(\Omega)$ such that, up to a subsequence, $\left(\left|u_{n}\right|^{p-2} u_{n}\right)$ is strongly convergent to $|u|^{p-2} u$ in $L^{r}(\Omega)$, whenever $r<\frac{N}{N-p}$

(c) if $u_{n}, u \in \Phi_{0}^{1, p}(\Omega),\left(u_{n}\right)$ is bounded in $\Phi_{0}^{1, p}(\Omega)$ and $\nabla u_{n} \rightarrow \nabla u$ a.e. in $\Omega$, then we have:

$$
\begin{aligned}
& \lim _{n}\left|\nabla u_{n}\right|^{p-2} \nabla u_{n}=|\nabla u|^{p-2} \nabla u \\
& \text { strongly in } L^{q}\left(\Omega ; \mathbb{R}^{N}\right), \text { for any } q<\frac{N}{N-1},
\end{aligned}
$$

(d) if $\left(u_{n}\right)$ is convergent to $u$ in $\Phi_{0}^{1, p}(\Omega)$, then we have

$$
\lim _{n} T_{k}\left(u_{n}\right)=T_{k}(u) \quad \text { strongly in } W_{0}^{1, p}(\Omega) \text {, for any } k>0 .
$$

Proof. The argument is an adaptation of the techniques of [5, 6, 23]. If $u \in \Phi_{0}^{1, p}(\Omega)$ and $p<N$, we have $\varphi_{p}(u) \in L^{p^{*}}(\Omega)$. Since $\varphi_{p}^{\prime}(s)$ behaves like

$$
\frac{1}{\left\{|s|(\log |s|)^{2}\right\}^{\frac{1}{p}}}
$$

at infinity, it follows that $|u|^{p-1} \in L^{r}(\Omega)$ whenever $r<\frac{N}{N-p}$.

If $q<\frac{p}{p-1}$, we also have

$$
\begin{aligned}
\int_{\Omega}|\nabla u|^{(p-1) q} d x & =\int_{\Omega}\left|\nabla\left[\varphi_{p}(u)\right]\right|^{(p-1) q} \frac{1}{\varphi_{p}^{\prime}(u)^{(p-1) q}} d x \\
& \leq\left(\int_{\Omega}\left|\nabla\left[\varphi_{p}(u)\right]\right|^{p} d x\right)^{\frac{(p-1) q}{p}}\left(\int_{\Omega} \frac{1}{\varphi_{p}^{\prime}(u)^{\frac{p(p-1) q}{p-(p-1) q}}} d x\right)^{\frac{p-(p-1) q}{p}} .
\end{aligned}
$$

In particular, if $q<\frac{N}{N-1}$ we also have

$$
\frac{p(p-1) q}{p-(p-1) q}<p(p-1) \frac{N}{N-p} .
$$

Taking into account the behavior of $\varphi_{p}^{\prime}$ at infinity and the previous assertion, we infer that $|\nabla u|^{p-1} \in L^{q}(\Omega)$. Therefore assertion $(a)$ is proved. 
The same argument shows that, if $\left(u_{n}\right)$ is bounded in $\Phi_{0}^{1, p}(\Omega)$, then $\left(\left|\nabla u_{n}\right|^{p-1}\right)$ is bounded in $L^{q}(\Omega)$ and $\left(\left|u_{n}\right|^{p-1}\right)$ is bounded in $L^{r}(\Omega)$, whenever $q<\frac{N}{N-1}$ and $r<\frac{N}{N-p}$. Moreover, up to a subsequence, $\left(\varphi_{p}\left(u_{n}\right)\right)$ is convergent to $\varphi_{p}(u)$ weakly in $W_{0}^{1, p}(\Omega)$ and a.e. in $\Omega$. It follows that $\left(u_{n}\right)$ is convergent to $u$ a.e. in $\Omega$, so that $\left(\left|u_{n}\right|^{p-2} u_{n}\right)$ is strongly convergent to $|u|^{p-2} u$ in $L^{r}(\Omega)$, whenever $r<\frac{N}{N-p}$. Therefore, assertion $(b)$ also holds. Then $(c)$ easily follows.

Finally, if $\left(u_{n}\right)$ is convergent to $u$ in $\Phi_{0}^{1, p}(\Omega)$, then $\left(\varphi_{p}\left(T_{k}\left(u_{n}\right)\right)\right)$ is strongly convergent to $\varphi_{p}\left(T_{k}(u)\right)$ in $W_{0}^{1, p}(\Omega)$, so that $\left(T_{k}\left(u_{n}\right)\right)$ is strongly convergent to $T_{k}(u)$ in $W_{0}^{1, p}(\Omega)$.

If $p=N$, the arguments are similar.

Finally, up to minor variants due to the presence of $\alpha_{0}$ in assumption $\left(a_{1}\right)$, the next regularity result can be proved as in $[5,6,23]$.

Theorem 2.10. If $\mu \in \mathcal{M}_{b}^{p}(\Omega)$ and $u$ is the entropy solution of $(2.1)$, then the following facts hold:

(a) if $\mu \in L^{m}(\Omega)$ with $1<m<\left(p^{*}\right)^{\prime}$, we have $|\nabla u|^{p-1} \in L^{m^{*}}(\Omega)$ and $|u|^{p-1} \in$ $L^{\frac{N m}{N-p m}}(\Omega)$;

(b) if $\mu \in \mathcal{M}_{b}^{p}(\Omega) \cap W^{-1, p^{\prime}}(\Omega)$, we have $u \in W_{0}^{1, p}(\Omega)$ and

$$
-\operatorname{div}[a(x, \nabla u)]=\mu \quad \text { in } W^{-1, p^{\prime}}(\Omega) .
$$

\section{A DEGREE FOR A CLASS OF QUASILINEAR ELLIPTIC EQUATIONS}

Consider again a bounded and open subset $\Omega$ of $\mathbb{R}^{N}$, a Carathéodory function $a: \Omega \times \mathbb{R}^{N} \rightarrow \mathbb{R}^{N}$ satisfying $\left(a_{1}\right)$ and $\left(a_{2}\right)$ and $\mu \in \mathcal{M}_{b}^{p}(\Omega)$. Let also

$$
b: \Omega \times\left(\mathbb{R} \times \mathbb{R}^{N}\right) \rightarrow \mathbb{R}
$$

be a Carathéodory function such that:

$\left(a_{3}\right)$ there exist $\alpha_{2} \in L^{1}(\Omega), \beta_{2} \in \mathbb{R}, 0<q<\frac{N(p-1)}{N-1}$ and $0<r<\frac{N(p-1)}{N-p}$ $(0<r<+\infty$ if $p=N)$ such that

$$
|b(x, s, \xi)| \leq \alpha_{2}(x)+\beta_{2}|s|^{r}+\beta_{2}|\xi|^{q}
$$

for a.e. $x \in \Omega$ and every $s \in \mathbb{R}$ and $\xi \in \mathbb{R}^{N}$.

By Proposition 2.9 and $\left(a_{3}\right)$, we have $b(x, u, \nabla u) \in L^{1}(\Omega)$ for any $u \in \Phi_{0}^{1, p}(\Omega)$. According to Remark 2.7, we say that $u$ is an entropy solution of

$$
\begin{cases}-\operatorname{div}[a(x, \nabla u)]+b(x, u, \nabla u)=\mu & \text { in } \Omega, \\ u=0 & \text { on } \partial \Omega,\end{cases}
$$

if $u \in \Phi_{0}^{1, p}(\Omega)$ and

$$
\begin{aligned}
\int_{\Omega} a(x, \nabla u) \cdot \nabla\left[T_{k}(u-v)\right] d x+ & \int_{\Omega} b(x, u, \nabla u) T_{k}(u-v) d x \\
& \leq \int_{\Omega} T_{k}(u-v) d \mu \quad \forall k>0, \forall v \in C_{c}^{\infty}(\Omega) .
\end{aligned}
$$

Remark 3.1. Let $u \in \Phi_{0}^{1, p}(\Omega)$ and let also $\hat{\mu} \in \mathcal{M}_{b}^{p}(\Omega)$ and

$$
\hat{a}: \Omega \times \mathbb{R}^{N} \rightarrow \mathbb{R}^{N}, \quad \hat{b}: \Omega \times\left(\mathbb{R} \times \mathbb{R}^{N}\right) \rightarrow \mathbb{R}
$$


be two Carathéodory functions satisfying $\left(a_{1}\right)-\left(a_{3}\right)$. Assume that

$$
\begin{aligned}
& \int_{\Omega}\{a(x, \nabla z) \cdot \nablav+b(x, z, \nabla z) v\} d x-\int_{\Omega} v d \mu \\
&=\int_{\Omega}\{\hat{a}(x, \nabla z) \cdot \nabla v+\hat{b}(x, z, \nabla z) v\} d x-\int_{\Omega} v d \hat{\mu} \\
& \forall z, v \in W_{0}^{1, p}(\Omega) \cap L^{\infty}(\Omega) .
\end{aligned}
$$

Then $u$ is an entropy solution of (3.1) if and only if $u$ is an entropy solution of

$$
\begin{cases}-\operatorname{div}[\hat{a}(x, \nabla u)]+\hat{b}(x, u, \nabla u)=\hat{\mu} & \text { in } \Omega, \\ u=0 & \text { on } \partial \Omega .\end{cases}
$$

Proof. For every $u \in \Phi_{0}^{1, p}(\Omega), v \in C_{c}^{\infty}(\Omega)$ and $h, k>0$, we have

$$
\begin{array}{r}
\int_{\Omega}\left\{a\left(x, \nabla\left[T_{h}(u)\right]\right) \cdot \nabla\left[T_{k}(u-v)\right]+b\left(x, T_{h}(u), \nabla\left[T_{h}(u)\right]\right) T_{k}(u-v)\right\} d x \\
-\int_{\Omega} T_{k}(u-v) d \mu \\
=\int_{\Omega}\left\{\hat{a}\left(x, \nabla\left[T_{h}(u)\right]\right) \cdot \nabla\left[T_{k}(u-v)\right]+\hat{b}\left(x, T_{h}(u), \nabla\left[T_{h}(u)\right]\right) T_{k}(u-v)\right\} d x \\
-\int_{\Omega} T_{k}(u-v) d \hat{\mu} .
\end{array}
$$

Passing to the limit as $h \rightarrow+\infty$, we get

$$
\begin{aligned}
& \int_{\Omega}\left\{a(x, \nabla u) \cdot \nabla\left[T_{k}(u-v)\right]+b(x, u, \nabla u) T_{k}(u-v)\right\} d x-\int_{\Omega} T_{k}(u-v) d \mu \\
& \quad=\int_{\Omega}\left\{\hat{a}(x, \nabla u) \cdot \nabla\left[T_{k}(u-v)\right]+\hat{b}(x, u, \nabla u) T_{k}(u-v)\right\} d x-\int_{\Omega} T_{k}(u-v) d \hat{\mu}
\end{aligned}
$$

and the assertion follows.

We will also consider parametric problems, in which $T$ is a metrizable topological space and

$$
a: \Omega \times\left(\mathbb{R}^{N} \times T\right) \rightarrow \mathbb{R}^{N}, \quad b: \Omega \times\left(\mathbb{R} \times \mathbb{R}^{N} \times T\right) \rightarrow \mathbb{R}
$$

are two Carathéodory functions satisfying $\left(a_{1}\right)-\left(a_{3}\right)$ uniformly, namely:

$\left(u_{1}\right)$ there exist $1<p \leq N, \alpha_{0} \in L^{1}(\Omega), \alpha_{1} \in L^{p^{\prime}}(\Omega), \beta_{1} \in \mathbb{R}$ and $\nu>0$ such that

$$
\begin{aligned}
& a_{t}(x, \xi) \cdot \xi \geq \nu|\xi|^{p}-\alpha_{0}(x), \\
& \left|a_{t}(x, \xi)\right| \leq \alpha_{1}(x)+\beta_{1}|\xi|^{p-1},
\end{aligned}
$$

for a.e. $x \in \Omega$ and every $\xi \in \mathbb{R}^{N}$ and $t \in T$;

$\left(u_{2}\right)$ we have

$$
\left[a_{t}(x, \xi)-a_{t}(x, \eta)\right] \cdot(\xi-\eta)>0
$$

for a.e. $x \in \Omega$ and every $\xi, \eta \in \mathbb{R}^{N}$ and $t \in T$ with $\xi \neq \eta$;

$\left(u_{3}\right)$ there exist $\alpha_{2} \in L^{1}(\Omega), \beta_{2} \in \mathbb{R}, 0<q<\frac{N(p-1)}{N-1}$ and $0<r<\frac{N(p-1)}{N-p}$ such that

$$
\left|b_{t}(x, s, \xi)\right| \leq \alpha_{2}(x)+\beta_{2}|s|^{r}+\beta_{2}|\xi|^{q}
$$


for a.e. $x \in \Omega$ and every $s \in \mathbb{R}, \xi \in \mathbb{R}^{N}$ and $t \in T$ (we write $a_{t}(x, \xi$ ), $b_{t}(x, s, \xi)$ instead of $\left.a(x,(\xi, t)), b(x,(s, \xi, t))\right)$.

In Section 7 we will see that it is possible to define a topological degree

$$
\operatorname{deg}(-\operatorname{div}[a(x, \nabla u)]+b(x, u, \nabla u), U, \mu) \in \mathbb{Z}
$$

whenever $U$ is a bounded and open subset of $\Phi_{0}^{1, p}(\Omega)$ such that (3.1) has no entropy solution $u \in \partial U$. We state here the main properties, referring to Section 7 for the proofs and further details.

Theorem 3.2. (Consistency property) Suppose that $\mu \in \mathcal{M}_{b}^{p}(\Omega) \cap W^{-1, p^{\prime}}(\Omega)$ and that $\alpha_{2} \in L^{1}(\Omega) \cap W^{-1, p^{\prime}}(\Omega)$ in assumption $\left(a_{3}\right)$.

Then the following facts hold:

(a) we have

$$
\left\{\begin{array}{l}
b(x, u, \nabla u) v \in L^{1}(\Omega) \\
b(x, u, \nabla u) \in L^{1}(\Omega) \cap W^{-1, p^{\prime}}(\Omega)
\end{array} \quad \text { for any } u, v \in W_{0}^{1, p}(\Omega)\right.
$$

and the map

$$
\begin{array}{ccc}
W_{0}^{1, p}(\Omega) & \longrightarrow & W^{-1, p^{\prime}}(\Omega) \\
u & \mapsto & -\operatorname{div}[a(x, \nabla u)]+b(x, u, \nabla u)
\end{array}
$$

is continuous and of class $(S)_{+}$;

(b) every entropy solution of (3.1) belongs to $W_{0}^{1, p}(\Omega)$ and every $u \in W_{0}^{1, p}(\Omega)$ is an entropy solution of (3.1) if and only if

$$
-\operatorname{div}[a(x, \nabla u)]+b(x, u, \nabla u)=\mu \quad \text { in } W^{-1, p^{\prime}}(\Omega) ;
$$

(c) if $U$ is a bounded and open subset of $\Phi_{0}^{1, p}(\Omega)$ such that (3.1) has no entropy solution $u \in \partial U$, then the set

$$
\{u \in U:-\operatorname{div}[a(x, \nabla u)]+b(x, u, \nabla u)=\mu\}
$$

is compact in $W_{0}^{1, p}(\Omega)$ and we have

$$
\begin{aligned}
\operatorname{deg}(-\operatorname{div}[a(x, \nabla u)] & +b(x, u, \nabla u), U, \mu) \\
& =\operatorname{deg}_{(S)_{+}}(-\operatorname{div}[a(x, \nabla u)]+b(x, u, \nabla u), U \cap V, \mu),
\end{aligned}
$$

whenever $V$ is a bounded and open subset of $W_{0}^{1, p}(\Omega)$ such that there are no solutions of (3.1) in $U \backslash V$ (we have denoted by $\operatorname{deg}_{(S)_{+}}$the degree for maps of class $(S)_{+}$as defined in $\left.[10,22,24]\right)$.

Theorem 3.3. (Normalization property) Let $\mu \in \mathcal{M}_{b}^{p}(\Omega)$ and let $U$ be any bounded and open subset of $\Phi_{0}^{1, p}(\Omega)$ containing the entropy solution $u$ of

$$
\begin{cases}-\operatorname{div}[a(x, \nabla u)]=\mu & \text { in } \Omega, \\ u=0 & \text { on } \partial \Omega .\end{cases}
$$

Then

$$
\operatorname{deg}(-\operatorname{div}[a(x, \nabla u)], U, \mu)=1 .
$$

Theorem 3.4. (Existence criterion) Let $\mu \in \mathcal{M}_{b}^{p}(\Omega)$ and let $U$ be a bounded and open subset of $\Phi_{0}^{1, p}(\Omega)$ such that (3.1) has no entropy solution $u \in \bar{U}$.

Then

$$
\operatorname{deg}(-\operatorname{div}[a(x, \nabla u)]+b(x, u, \nabla u), U, \mu)=0 .
$$


Theorem 3.5. (Additivity property) Let $\mu \in \mathcal{M}_{b}^{p}(\Omega)$ and let $U$ be a bounded and open subset of $\Phi_{0}^{1, p}(\Omega)$ such that (3.1) has no entropy solution $u \in \partial U$. Assume that $U=U_{1} \cup U_{2}$, where $U_{1}, U_{2}$ are two disjoint open subsets of $\Phi_{0}^{1, p}(\Omega)$.

Then

$$
\begin{aligned}
\operatorname{deg}(-\operatorname{div}[a(x, \nabla u)] & +b(x, u, \nabla u), U, \mu) \\
=\operatorname{deg}(-\operatorname{div}[a(x, & \left.\nabla u)]+b(x, u, \nabla u), U_{1}, \mu\right) \\
& +\operatorname{deg}\left(-\operatorname{div}[a(x, \nabla u)]+b(x, u, \nabla u), U_{2}, \mu\right) .
\end{aligned}
$$

Theorem 3.6. (Excision property) Let $\mu \in \mathcal{M}_{b}^{p}(\Omega)$ and let $V \subseteq U$ be two bounded and open subsets of $\Phi_{0}^{1, p}(\Omega)$ such that (3.1) has no entropy solution $u \in$ $\bar{U} \backslash V$.

Then

$$
\begin{array}{r}
\operatorname{deg}(-\operatorname{div}[a(x, \nabla u)]+b(x, u, \nabla u), U, \mu) \\
\quad=\operatorname{deg}(-\operatorname{div}[a(x, \nabla u)]+b(x, u, \nabla u), V, \mu) .
\end{array}
$$

Theorem 3.7. (Homotopy invariance property) Let

$$
a: \Omega \times\left(\mathbb{R}^{N} \times[0,1]\right) \rightarrow \mathbb{R}^{N}, \quad b: \Omega \times\left(\mathbb{R} \times \mathbb{R}^{N} \times[0,1]\right) \rightarrow \mathbb{R}
$$

be two Carathéodory functions satisfying $\left(u_{1}\right)-\left(u_{3}\right)$ with respect to $T=[0,1]$ and let $\mu_{0}, \mu_{1} \in \mathcal{M}_{b}^{p}(\Omega)$.

Then the following facts hold:

(a) for every bounded and closed subset $C$ of $\Phi_{0}^{1, p}(\Omega)$, the set of $t$ 's in $[0,1]$ such that

$$
\begin{cases}-\operatorname{div}\left[a_{t}(x, \nabla u)\right]+b_{t}(x, u, \nabla u)=(1-t) \mu_{0}+t \mu_{1} & \text { in } \Omega, \\ u=0 & \text { on } \partial \Omega,\end{cases}
$$

admits an entropy solution $u \in C$ is closed in $[0,1]$;

(b) for every bounded and open subset $U$ of $\Phi_{0}^{1, p}(\Omega)$, if (3.2) has no entropy solution with $t \in[0,1]$ and $u \in \partial U$, then

$$
\operatorname{deg}\left(-\operatorname{div}\left[a_{t}(x, \nabla u)\right]+b_{t}(x, u, \nabla u), U,(1-t) \mu_{0}+t \mu_{1}\right)
$$

is independent of $t \in[0,1]$.

Theorem 3.8. Let $\mu=0$ and let $U$ be a bounded and open subset of $\Phi_{0}^{1, p}(\Omega)$ such that (3.1) has no entropy solution $u \in \partial U$. Assume that $U$ is symmetric with $0 \in U$ and that

$$
a(x,-\xi)=-a(x, \xi), b(x,-s,-\xi)=-b(x, s, \xi),
$$

for a.e. $x \in \Omega$ and every $s \in \mathbb{R}$ and $\xi \in \mathbb{R}^{N}$.

Then

$$
\operatorname{deg}(-\operatorname{div}[a(x, \nabla u)]+b(x, u, \nabla u), U, 0)
$$

is an odd integer. 


\section{Proof of Theorem 1.1}

Lemma 4.1. Let

$$
a: \Omega \times \mathbb{R}^{N} \rightarrow \mathbb{R}^{N}, \quad b: \Omega \times\left(\mathbb{R} \times \mathbb{R}^{N}\right) \rightarrow \mathbb{R}
$$

be two Carathéodory functions satisfying $(i)-(i i i)$.

Then the following facts hold:

(a) if we set

$$
\begin{aligned}
& a_{t}(x, \xi)= \begin{cases}t a\left(x, t^{-\frac{1}{p-1}} \xi\right) & \text { if } 0<t \leq 1, \\
a_{\infty}(x, \xi) & \text { if } t=0,\end{cases} \\
& b_{t}(x, s, \xi)= \begin{cases}t b\left(x, t^{-\frac{1}{p-1}} s, t^{-\frac{1}{p-1}} \xi\right) & \text { if } 0<t \leq 1, \\
b_{\infty}(x, s, \xi) & \text { if } t=0,\end{cases}
\end{aligned}
$$

then $a_{t}, b_{t}$ are two Carathéodory functions satisfying $(i),($ ii) uniformly for $t \in[0,1]$, namely

$$
\begin{aligned}
& a_{t}(x, \xi) \cdot \xi \geq \nu|\xi|^{p}-\alpha_{0}(x), \\
& \left|a_{t}(x, \xi)\right| \leq \alpha_{1}(x)+\beta|\xi|^{p-1}, \\
& \left|b_{t}(x, s, \xi)\right| \leq \alpha_{2}(x)+\beta|s|^{p-1}+\beta|\xi|^{p-1}, \\
& {\left[a_{t}(x, \xi)-a_{t}(x, \eta)\right] \cdot(\xi-\eta)>0,}
\end{aligned}
$$

for a.e. $x \in \Omega$ and every $s \in \mathbb{R}, \xi, \eta \in \mathbb{R}^{N}, t \in[0,1]$ with $\xi \neq \eta$; in particular, $a_{t}, b_{t}$ satisfy $\left(u_{1}\right)-\left(u_{3}\right)$ with respect to $T=[0,1]$;

(b) if the problem

$$
\left\{\begin{array}{l}
u \in W_{0}^{1, p}(\Omega) \backslash\{0\}, \\
-\operatorname{div}\left[a_{\infty}(x, \nabla u)\right]+b_{\infty}(x, u, \nabla u)=0 \quad \text { in } W^{-1, p^{\prime}}(\Omega)
\end{array}\right.
$$

has no solution then, for every $\mu \in \mathcal{M}_{b}^{p}(\Omega)$, there exists $R>0$ such that the problem

$$
\begin{cases}-\operatorname{div}\left[a_{t}(x, \nabla u)\right]+b_{t}(x, u, \nabla u)=t \mu & \text { in } \Omega, \\ u=0 & \text { on } \partial \Omega\end{cases}
$$

has no entropy solution with $0 \leq t \leq 1$ and $u \in \Phi_{0}^{1, p}(\Omega)$ with

$$
\int_{\Omega}\left|\nabla\left[\varphi_{p}(u)\right]\right|^{p} d x \geq R
$$

Proof. Assertion ( $a$ ) is easy to prove. To prove assertion (b), assume for a contradiction that $\left(u_{k}, t_{k}\right)$ is a sequence of solutions of (4.5) with $0 \leq t_{k} \leq 1, u_{k} \in \Phi_{0}^{1, p}(\Omega)$ and

$$
\int_{\Omega}\left|\nabla\left[\varphi_{p}\left(u_{k}\right)\right]\right|^{p} d x \geq k .
$$

By (4.1) and (2.5), it follows

$$
\lim _{k} \int_{\Omega}\left|b_{t_{k}}\left(x, u_{k}, \nabla u_{k}\right)\right| d x=+\infty,
$$


whence

$$
\lim _{k} \int_{\Omega}\left(\left|u_{k}\right|^{p-1}+\left|\nabla u_{k}\right|^{p-1}\right) d x=+\infty
$$

by (4.3). If we set $u_{k}=\tau_{k}^{\frac{1}{p-1}} v_{k}, \sigma_{k}=t_{k} / \tau_{k}$ with

$$
\begin{gathered}
\tau_{k}=\int_{\Omega}\left(\left|u_{k}\right|^{p-1}+\left|\nabla u_{k}\right|^{p-1}\right) d x, \\
\int_{\Omega}\left(\left|v_{k}\right|^{p-1}+\left|\nabla v_{k}\right|^{p-1}\right) d x=1,
\end{gathered}
$$

it follows that $\left(v_{k}, \sigma_{k}\right)$ satisfies

$$
\begin{cases}-\operatorname{div}\left[a_{\sigma_{k}}\left(x, \nabla v_{k}\right)\right]+b_{\sigma_{k}}\left(x, v_{k}, \nabla v_{k}\right)=\sigma_{k} \mu & \text { in } \Omega, \\ v_{k}=0 & \text { on } \partial \Omega\end{cases}
$$

with $\sigma_{k} \rightarrow 0$. By (4.7) and Proposition 2.9 we infer that

$$
\inf _{k} \int_{\Omega}\left|\nabla\left[\varphi_{p}\left(v_{k}\right)\right]\right|^{p} d x>0
$$

while (4.7), (4.3), (4.1) and (2.5) imply that

$$
\sup _{k} \int_{\Omega}\left|\nabla\left[\varphi_{p}\left(v_{k}\right)\right]\right|^{p} d x<+\infty
$$

If we set

$$
C=\left\{u \in \Phi_{0}^{1, p}(\Omega): \frac{1}{M} \leq \int_{\Omega}\left|\nabla\left[\varphi_{p}(u)\right]\right|^{p} d x \leq M\right\},
$$

where $M>0$ satisfies

$$
\frac{1}{M} \leq \int_{\Omega}\left|\nabla\left[\varphi_{p}\left(v_{k}\right)\right]\right|^{p} d x \leq M \quad \forall k \in \mathbb{N},
$$

we have that $C$ is a bounded and closed subset of $\Phi_{0}^{1, p}(\Omega)$. By $(a)$ of Theorem 3.7, there exists an entropy solution $v \in C \subseteq \Phi_{0}^{1, p}(\Omega) \backslash\{0\}$ of

$$
\begin{cases}-\operatorname{div}\left[a_{0}(x, \nabla v)\right]+b_{0}(x, v, \nabla v)=0 & \text { in } \Omega, \\ v=0 & \text { on } \partial \Omega .\end{cases}
$$

Because of (1.4), we have that $b_{0}$ satisfies $\left(a_{3}\right)$ with $\alpha_{2} \in L^{\infty}(\Omega)$. From Theorem 3.2 we infer that $v$ satisfies

$$
\left\{\begin{array}{l}
v \in W_{0}^{1, p}(\Omega) \backslash\{0\} \\
-\operatorname{div}\left[a_{\infty}(x, \nabla v)\right]+b_{\infty}(x, v, \nabla v)=0 \quad \text { in } W^{-1, p^{\prime}}(\Omega),
\end{array}\right.
$$

and a contradiction follows.

\section{Proof of Theorem 1.1.}

Let $a_{t}, b_{t}$ be as in Lemma 4.1. Assume that assertion $(a)$ of Theorem 1.1 is false and take $\mu \in \mathcal{M}_{b}^{p}(\Omega)$. Then there exists $R>0$ such that (4.5) has no entropy solution with $0 \leq t \leq 1$ and $u \in \Phi_{0}^{1, p}(\Omega)$ with

$$
\int_{\Omega}\left|\nabla\left[\varphi_{p}(u)\right]\right|^{p} d x \geq R
$$

If we set

$$
U=\left\{u \in \Phi_{0}^{1, p}(\Omega): \int_{\Omega}\left|\nabla\left[\varphi_{p}(u)\right]\right|^{p} d x<R\right\}
$$


from $(b)$ of Theorem 3.7 we infer that

$$
\begin{aligned}
\operatorname{deg}(-\operatorname{div}[a(x, \nabla u)]+b(x, u, \nabla u) & , U, \mu) \\
& =\operatorname{deg}\left(-\operatorname{div}\left[a_{\infty}(x, \nabla u)\right]+b_{\infty}(x, u, \nabla u), U, 0\right) .
\end{aligned}
$$

On the other hand,

$$
\operatorname{deg}\left(-\operatorname{div}\left[a_{\infty}(x, \nabla u)\right]+b_{\infty}(x, u, \nabla u), U, 0\right)
$$

is an odd integer by (1.7) and Theorem 3.8, whence

$$
\operatorname{deg}(-\operatorname{div}[a(x, \nabla u)]+b(x, u, \nabla u), U, \mu) \neq 0 .
$$

By Theorem 3.4 there exists an entropy solution $u \in U$ of

$$
\begin{cases}-\operatorname{div}[a(x, \nabla u)]+b(x, u, \nabla u)=\mu & \text { in } \Omega, \\ u=0 & \text { on } \partial \Omega\end{cases}
$$

and assertion $(b)$ of Theorem 1.1 follows.

\section{ENTROPY SOLUTIONS OF PARAMETRIC PROBLEMS}

Throughout this section, we consider a metrizable topological space $T$, two Carathéodory functions

$$
a: \Omega \times\left(\mathbb{R}^{n} \times T\right) \rightarrow \mathbb{R}^{n}, \quad b: \Omega \times\left(\mathbb{R} \times \mathbb{R}^{n} \times T\right) \rightarrow \mathbb{R}
$$

satisfying $\left(u_{1}\right)-\left(u_{3}\right)$ and the entropy solutions of

$$
\begin{cases}-\operatorname{div}\left[a_{t}(x, \nabla u)\right]+b_{t}(x, u, \nabla u)=\mu & \text { in } \Omega, \\ u=0 & \text { on } \partial \Omega,\end{cases}
$$

with $\mu \in \mathcal{M}_{b}^{p}(\Omega)$.

Theorem 5.1. Let $\left(t_{n}\right)$ be a sequence in $T,\left(\mu_{n}\right)$ a sequence in $\mathcal{M}_{b}^{p}(\Omega)$ and $\left(u_{n}\right)$ a sequence of entropy solutions of (5.1) associated with $t_{n}$ and $\mu_{n}$. Let also $t \in T$ and $\mu \in \mathcal{M}_{b}^{p}(\Omega)$. Assume that $\left(u_{n}\right)$ is bounded in $\Phi_{0}^{1, p}(\Omega),\left(t_{n}\right)$ is convergent to $t$ in $T$ and there exist $\left(w_{n}^{(0)}\right), w^{(0)}$ in $L^{1}(\Omega)$ and $\left(w_{n}^{(1)}\right), w^{(1)}$ in $L^{p^{\prime}}\left(\Omega ; \mathbb{R}^{N}\right)$ such that

$$
\begin{gathered}
\int_{\Omega} v d \mu_{n}=\int_{\Omega} v w_{n}^{(0)} d x+\int_{\Omega}(\nabla v) \cdot w_{n}^{(1)} d x, \\
\int_{\Omega} v d \mu=\int_{\Omega} v w^{(0)} d x+\int_{\Omega}(\nabla v) \cdot w^{(1)} d x, \\
\forall v \in W_{0}^{1, p}(\Omega) \cap L^{\infty}(\Omega),
\end{gathered}
$$

and such that $\left(w_{n}^{(0)}\right)$ is weakly convergent to $w^{(0)}$ in $L^{1}(\Omega)$, while $\left(w_{n}^{(1)}\right)$ is strongly convergent to $w^{(1)}$ in $L^{p^{\prime}}\left(\Omega ; \mathbb{R}^{N}\right)$.

Then there exists a subsequence $\left(u_{n_{j}}\right)$ converging in $\Phi_{0}^{1, p}(\Omega)$ to an entropy solution $u$ of (5.1) associated with $t$ and $\mu$.

Corollary 5.2. Let $C$ be a bounded and closed subset of $\Phi_{0}^{1, p}(\Omega)$ and let $\mu \in$ $\mathcal{M}_{b}^{p}(\Omega)$. Then the set

$$
\{t \in T:(5.1) \text { admits an entropy solution } u \in C\}
$$

is closed in $T$.

Proof. It is an obvious consequence of Theorem 5.1. 
The proof of Theorem 5.1 will be given at the end of the section. The next lemma is an adaptation of results of $[3,7,20]$ and concerns the entropy solutions of

with $\mu \in \mathcal{M}_{b}^{p}(\Omega)$.

$$
\begin{cases}-\operatorname{div}\left[a_{t}(x, \nabla u)\right]=\mu & \text { in } \Omega \\ u=0 & \text { on } \partial \Omega\end{cases}
$$

Lemma 5.3. Let $\left(t_{n}\right)$ be a sequence in $T,\left(\mu_{n}\right)$ a sequence in $\mathcal{M}_{b}^{p}(\Omega)$ and let $\left(u_{n}\right)$ be the entropy solution of (5.2) associated with $t_{n}$ and $\mu_{n}$. Assume that $\left(t_{n}\right)$ is convergent in $T$ and there exist two sequences $\left(w_{n}^{(0)}\right)$ in $L^{1}(\Omega)$ and $\left(w_{n}^{(1)}\right)$ in $L^{p^{\prime}}\left(\Omega ; \mathbb{R}^{N}\right)$ such that

$$
\int_{\Omega} v d \mu_{n}=\int_{\Omega} v w_{n}^{(0)} d x+\int_{\Omega}(\nabla v) \cdot w_{n}^{(1)} d x \quad \forall v \in W_{0}^{1, p}(\Omega) \cap L^{\infty}(\Omega),
$$

and such that $\left(w_{n}^{(0)}\right)$ bounded in $L^{1}(\Omega)$, while $\left(w_{n}^{(1)}\right)$ is strongly convergent in $L^{p^{\prime}}\left(\Omega ; \mathbb{R}^{N}\right)$.

Then $\left(u_{n}\right)$ is bounded in $\Phi_{0}^{1, p}(\Omega)$ and there exist $u \in \Phi_{0}^{1, p}(\Omega)$ and a subsequence $\left(u_{n_{j}}\right)$ such that $\left(u_{n_{j}}, \nabla u_{n_{j}}\right)$ is convergent to $(u, \nabla u)$ a.e. in $\Omega$.

Proof. If we set

$$
\tilde{a}_{n}(x, \xi)=a_{t_{n}}(x, \xi)-w_{n}^{(1)}
$$

it is easily seen that each $\tilde{a}_{n}$ satisfies $\left(a_{1}\right)-\left(a_{2}\right)$ for some $\alpha_{0}, \alpha_{1}, \beta_{1}$ and $\nu$ independent of $n$. Since $u_{n}$ is an entropy solution of

$$
\begin{cases}-\operatorname{div}\left[\tilde{a}_{n}\left(x, \nabla u_{n}\right)\right]=w_{n}^{(0)} & \text { in } \Omega, \\ u=0 & \text { on } \partial \Omega,\end{cases}
$$

by $(2.5)\left(u_{n}\right)$ is bounded in $\Phi_{0}^{1, p}(\Omega)$.

Therefore there exist $u \in \Phi_{0}^{1, p}(\Omega)$ and a subsequence $\left(u_{n_{j}}\right)$ such that $\left(\varphi_{p}\left(u_{n_{j}}\right)\right)$ is convergent to $\varphi_{p}(u)$ weakly in $W_{0}^{1, p}(\Omega)$ and $\left(u_{n_{j}}\right)$ is convergent to $u$ a.e. in $\Omega$. It follows that $\left(T_{k}\left(u_{n_{j}}\right)\right)$ is weakly convergent to $T_{k}(u)$ in $W_{0}^{1, p}(\Omega)$.

Now let $\vartheta: \mathbb{R} \rightarrow[0,1]$ be a $C^{\infty}$-function such that $\vartheta(s)=1$ for $|s| \leq 1$ and $\vartheta(s)=0$ for $|s| \geq 2$. By Theorem 2.5, we have

$$
\begin{aligned}
\int_{\Omega} a_{t_{n}} & \left(x, \nabla u_{n}\right) \cdot \nabla\left[\vartheta\left(\frac{u_{n}}{k}\right) v\right] d x \\
& =\int_{\Omega}\left[\vartheta\left(\frac{u_{n}}{k}\right) v\right] w_{n}^{(0)} d x+\int_{\Omega} \nabla\left[\vartheta\left(\frac{u_{n}}{k}\right) v\right] \cdot w_{n}^{(1)} d x \quad \forall v \in C_{c}^{\infty}(\Omega),
\end{aligned}
$$

namely

$$
\int_{\Omega} \hat{a}_{n}\left(x, \nabla T_{2 k}\left(u_{n}\right)\right) \cdot \nabla v d x=\int_{\Omega} v \hat{w}_{n}^{(0)} d x+\int_{\Omega} \nabla v \cdot \hat{w}_{n}^{(1)} d x \quad \forall v \in C_{c}^{\infty}(\Omega),
$$

where

$$
\begin{aligned}
& \hat{a}_{n}(x, \xi)=\vartheta\left(\frac{u_{n}(x)}{k}\right) a_{t_{n}}(x, \xi) \\
& \hat{w}_{n}^{(0)}=\vartheta\left(\frac{u_{n}}{k}\right) w_{n}^{(0)}-\vartheta^{\prime}\left(\frac{u_{n}}{k}\right) \frac{\left[a_{t_{n}}\left(x, \nabla T_{2 k}\left(u_{n}\right)\right)-w_{n}^{(1)}\right] \cdot \nabla T_{2 k}\left(u_{n}\right)}{k} \\
& \hat{w}_{n}^{(1)}=\vartheta\left(\frac{u_{n}}{k}\right) w_{n}^{(1)} .
\end{aligned}
$$


If we also set

$$
\begin{aligned}
& \hat{a}(x, \xi)=\vartheta\left(\frac{u(x)}{k}\right) a_{t}(x, \xi), \\
& E_{k}=\{x \in \Omega:|u(x)|<k\},
\end{aligned}
$$

we can apply [11, Theorem 1]. Therefore, for any $k>0$, up to a further subsequence $\left(\nabla u_{n_{j}}\right)$ is convergent to $\nabla u$ a.e. in $E_{k}$. A standard diagonal argument shows that, up to another subsequence, $\left(\nabla u_{n_{j}}\right)$ is convergent to $\nabla u$ a.e. in $\Omega$.

Lemma 5.4. Let $\left(t_{n}\right), t$ be in $T$ and $\left(\mu_{n}\right), \mu$ in $\mathcal{M}_{b}^{p}(\Omega)$. Let also $\left(u_{n}\right)$, $u$ be the entropy solutions of (5.2) associated with $t_{n}, \mu_{n}$ and $t, \mu$, respectively. Assume that $\left(t_{n}\right)$ is convergent to $t$ in $T$ and there exist $\left(w_{n}^{(0)}\right), w^{(0)}$ in $L^{1}(\Omega)$ and $\left(w_{n}^{(1)}\right)$, $w^{(1)}$ in $L^{p^{\prime}}\left(\Omega ; \mathbb{R}^{N}\right)$ such that

$$
\begin{aligned}
\int_{\Omega} v d \mu_{n}=\int_{\Omega} v w_{n}^{(0)} d x+\int_{\Omega}(\nabla v) \cdot w_{n}^{(1)} d x \\
\int_{\Omega} v d \mu=\int_{\Omega} v w^{(0)} d x+\int_{\Omega}(\nabla v) \cdot w^{(1)} d x,
\end{aligned}
$$

$\forall v \in W_{0}^{1, p}(\Omega) \cap L^{\infty}(\Omega)$,

and such that $\left(w_{n}^{(0)}\right)$ is weakly convergent to $w^{(0)}$ in $L^{1}(\Omega)$, while $\left(w_{n}^{(1)}\right)$ is strongly convergent to $w^{(1)}$ in $L^{p^{\prime}}\left(\Omega ; \mathbb{R}^{N}\right)$.

Then $\left(u_{n}\right)$ is convergent to $u$ in $\Phi_{0}^{1, p}(\Omega)$.

Proof. First of all, by Lemma $5.3\left(u_{n}\right)$ is bounded in $\Phi_{0}^{1, p}(\Omega)$ and there exists $\hat{u} \in \Phi_{0}^{1, p}(\Omega)$ such that, up to a subsequence, $\left(u_{n}, \nabla u_{n}\right)$ is convergent to $(\hat{u}, \nabla \hat{u})$ a.e. in $\Omega$. Since $\left(\varphi_{p}\left(u_{n}\right)\right)$ is weakly convergent to $\varphi_{p}(\hat{u})$ in $W_{0}^{1, p}(\Omega)$, it follows that $\left(\nabla\left[T_{k}\left(u_{n}\right)\right]\right)$ is convergent to $\nabla\left[T_{k}(\hat{u})\right]$ weakly in $L^{p}\left(\Omega ; \mathbb{R}^{N}\right)$ and a.e. in $\Omega$, for any $k>0$. For any $k>0$ and $v \in C_{c}^{\infty}(\Omega)$, it follows that

$$
\begin{array}{r}
\lim _{n} \int_{\Omega} T_{k}\left(u_{n}-v\right) d \mu_{n}=\lim _{n} \int_{\Omega}\left(T_{k}\left(u_{n}-v\right) w_{n}^{(0)}+\nabla\left[T_{k}\left(u_{n}-v\right)\right] \cdot w_{n}^{(1)}\right) d x \\
=\int_{\Omega}\left(T_{k}(\hat{u}-v) w^{(0)}+\nabla\left[T_{k}(\hat{u}-v)\right] \cdot w^{(1)}\right) d x=\int_{\Omega} T_{k}(\hat{u}-v) d \mu .
\end{array}
$$

On the other hand, if $v \in C_{c}^{\infty}(\Omega)$ and $h=k+\|v\|_{\infty}$, we have

$$
\begin{aligned}
\int_{\Omega} T_{k}\left(u_{n}-v\right) d \mu_{n} & \geq \int_{\Omega} a_{t_{n}}\left(x, \nabla u_{n}\right) \cdot \nabla\left[T_{k}\left(u_{n}-v\right)\right] d x \\
& =\int_{\left\{\left|u_{n}-v\right|<k\right\}} a_{t_{n}}\left(x, \nabla T_{h}\left(u_{n}\right)\right) \cdot \nabla T_{h}\left(u_{n}\right) d x \\
& -\int_{\left\{\left|u_{n}-v\right|<k\right\}} a\left(x, \nabla T_{h}\left(u_{n}\right)\right) \cdot \nabla v d x .
\end{aligned}
$$

Since $a_{t_{n}}\left(x, \nabla T_{h}\left(u_{n}\right)\right) \cdot \nabla T_{h}\left(u_{n}\right) \geq-\alpha_{0}$, we can pass to the lower limit as $n \rightarrow \infty$ and apply Fatou's lemma, obtaining

$$
\int_{\Omega} T_{k}(\hat{u}-v) d \mu \geq \int_{\Omega} a_{t}(x, \nabla \hat{u}) \cdot \nabla\left[T_{k}(\hat{u}-v)\right] d x \quad \forall v \in C_{c}^{\infty}(\Omega) .
$$

By the uniqueness of the entropy solution we infer that $\hat{u}=u$. 
According to Theorem 2.6, we also have

$$
\begin{aligned}
\int_{\Omega} \psi^{\prime}\left(u_{n}\right) a_{t_{n}}\left(x, \nabla u_{n}\right) \cdot \nabla u_{n} d x & =\int_{\Omega} \psi\left(u_{n}\right) d \mu_{n}, \\
\int_{\Omega} \psi^{\prime}(u) a_{t}(x, \nabla u) \cdot \nabla u d x & =\int_{\Omega} \psi(u) d \mu .
\end{aligned}
$$

Since

$$
\psi^{\prime}\left(u_{n}\right) a_{t_{n}}\left(x, \nabla u_{n}\right) \cdot \nabla u_{n}-\nu\left|\nabla\left[\varphi_{p}\left(u_{n}\right)\right]\right|^{p} \geq-\alpha_{0},
$$

again from Fatou's lemma we infer that

$$
\begin{aligned}
& \int_{\Omega} \psi(u) d \mu-\nu \int_{\Omega}\left|\nabla\left[\varphi_{p}(u)\right]\right|^{p} d x=\int_{\Omega} \psi^{\prime}(u) a_{t}(x, \nabla u) \cdot \nabla u d x \\
& -\nu \int_{\Omega}\left|\nabla\left[\varphi_{p}(u)\right]\right|^{p} d x \\
& \leq \limsup _{n} \int_{\Omega} \psi^{\prime}\left(u_{n}\right) a_{t_{n}}\left(x, \nabla u_{n}\right) \cdot \nabla u_{n} d x \\
& -\nu \limsup _{n} \int_{\Omega}\left|\nabla\left[\varphi_{p}\left(u_{n}\right)\right]\right|^{p} d x \\
& =\limsup _{n} \int_{\Omega} \psi\left(u_{n}\right) d \mu_{n} \\
& -\nu \limsup _{n} \int_{\Omega}\left|\nabla\left[\varphi_{p}\left(u_{n}\right)\right]\right|^{p} d x \\
& =\int_{\Omega} \psi(u) d \mu \\
& -\nu \limsup \int_{\Omega}\left|\nabla\left[\varphi_{p}\left(u_{n}\right)\right]\right|^{p} d x .
\end{aligned}
$$

It follows

$$
\limsup _{n} \int_{\Omega}\left|\nabla\left[\varphi_{p}\left(u_{n}\right)\right]\right|^{p} d x \leq \int_{\Omega}\left|\nabla\left[\varphi_{p}(u)\right]\right|^{p} d x,
$$

whence the strong convergence of $\left(u_{n}\right)$ to $u$ in $\Phi_{0}^{1, p}(\Omega)$.

Proof of Theorem 5.1.

Since $\left(u_{n}\right)$ is bounded in $\Phi_{0}^{1, p}(\Omega)$, from $(b)$ of Proposition 2.9 and $\left(u_{3}\right)$ it follows that $\left(b_{t_{n}}\left(x, u_{n}, \nabla u_{n}\right)\right)$ is bounded in $L^{1}(\Omega)$. By Lemma 5.3 we infer that there exists $u \in \Phi_{0}^{1, p}(\Omega)$ such that, up to a subsequence, $\left(u_{n}, \nabla u_{n}\right)$ is convergent to $(u, \nabla u)$ a.e. in $\Omega$.

From Proposition 2.9 and $\left(u_{3}\right)$ we deduce that $\left(b_{t_{n}}\left(x, u_{n}, \nabla u_{n}\right)\right)$ is (strongly) convergent to $b_{t}(x, u, \nabla u)$ in $L^{1}(\Omega)$. By Lemma 5.4 we conclude that $\left(u_{n}\right)$ is convergent to $u$ in $\Phi_{0}^{1, p}(\Omega)$ and that $u$ is an entropy solution of (5.1).

\section{Degree theory in Reflexive Banach spaces}

Let $X$ be a reflexive real Banach space.

Definition 6.1. A map $F: D \rightarrow X^{\prime}$, with $D \subseteq X$, is said to be of class $(S)_{+}$if, for every sequence $\left(u_{n}\right)$ in $D$ weakly converging to some $u$ in $X$ with

$$
\limsup _{n}\left\langle F\left(u_{n}\right), u_{n}-u\right\rangle \leq 0,
$$

it holds $\left\|u_{n}-u\right\| \rightarrow 0$. 
More generally, if $T$ is a metrizable topological space, a map $H: D \rightarrow X^{\prime}$, with $D \subseteq X \times T$, is said to be of class $(S)_{+}$if, for every sequence $\left(u_{n}, t_{n}\right)$ in $D$ with $\left(u_{n}\right)$ weakly converging to $u$ in $X,\left(t_{n}\right)$ converging to $t$ in $T$ and

$$
\limsup \left\langle H_{t_{n}}\left(u_{n}\right), u_{n}-u\right\rangle \leq 0
$$

it holds $\left\|u_{n}-u\right\| \rightarrow 0$ (we write $H_{t}(u)$ instead of $H(u, t)$ ).

Assume now that $U$ is a bounded and open subset of $X, F: \bar{U} \rightarrow X^{\prime}$ a continuous map of class $(S)_{+}$and $w \in X^{\prime}$.

Remark 6.2. It is easily seen that the set

$$
\{u \in \bar{U}: F(u)=w\}
$$

is compact (possibly empty).

According to $[10,22,24]$, if $w \notin F(\partial U)$, one can define the topological degree

$$
\operatorname{deg}_{(S)_{+}}(F, U, w) \in \mathbb{Z}
$$

Let us recall some basic properties.

Proposition 6.3. If $w \notin F(\partial U)$, then

$$
\operatorname{deg}_{(S)_{+}}(F, U, w)=\operatorname{deg}_{(S)_{+}}(F-w, U, 0) .
$$

Theorem 6.4. If $w \notin F(\partial U), u_{0} \in U$ and

$$
\left\langle F(u), u-u_{0}\right\rangle \geq\left\langle w, u-u_{0}\right\rangle \quad \text { for any } u \in \partial U,
$$

then $\operatorname{deg}_{(S)_{+}}(F, U, w)=1$.

Theorem 6.5. If $w \notin F(\bar{U})$, then $\operatorname{deg}_{(S)_{+}}(F, U, w)=0$.

Theorem 6.6. If $w \notin F(\partial U)$ and $U=U_{0} \cup U_{1}$, where $U_{0}, U_{1}$ are two disjoint open subsets of $X$, then

$$
\operatorname{deg}_{(S)_{+}}(F, U, w)=\operatorname{deg}_{(S)_{+}}\left(F, U_{0}, w\right)+\operatorname{deg}_{(S)_{+}}\left(F, U_{1}, w\right) .
$$

Theorem 6.7. Let $V$ be another open subset of $X$ with $V \subseteq U$ and assume that $w \notin F(\bar{U} \backslash V)$.

Then $\operatorname{deg}_{(S)_{+}}(F, U, w)=\operatorname{deg}_{(S)_{+}}(F, V, w)$.

Theorem 6.8. Let $H: \bar{U} \times[0,1] \rightarrow X^{\prime}$ be a continuous map of class $(S)_{+}$. Then the following facts hold:

(a) the set

$$
\left\{(u, t) \in \bar{U} \times[0,1]: H_{t}(u)=w\right\}
$$

is compact (possibly empty);

(b) if $w \notin H(\partial U \times[0,1])$, then $\operatorname{deg}_{(S)_{+}}\left(H_{t}, U, w\right)$ is independent of $t \in[0,1]$.

Theorem 6.9. Assume that $U$ is symmetric with $0 \in U$ and that $F$ is odd with $0 \notin F(\partial U)$.

Then $\operatorname{deg}_{(S)_{+}}(F, U, 0)$ is an odd integer.

Let us also introduce a variant, more in the line of the degree for "compactly rooted maps" of [14].

Assume that $U$ is a (possibly unbounded) open subset of $X, F: U \rightarrow X^{\prime}$ is continuous and locally of class $(S)_{+}$and $w \in X^{\prime}$. 
Proposition 6.10. If

$$
\{u \in U: F(u)=w\} \text { is compact (possibly empty), }
$$

then the following facts hold:

(a) there exists a bounded and open subset $V$ of $X$ such that $\bar{V} \subseteq U, F$ is of class $(S)_{+}$on $\bar{V}$ and $w \notin F(U \backslash V)$;

(b) if $V_{0}$ and $V_{1}$ are as in $(a)$, it holds

$$
\operatorname{deg}_{(S)_{+}}\left(F, V_{0}, w\right)=\operatorname{deg}_{(S)_{+}}\left(F, V_{1}, w\right) .
$$

Proof. Assertion $(a)$ is easy to prove. If $V_{0}$ and $V_{1}$ are as in $(a)$, from Theorem 6.7 we infer that

$$
\operatorname{deg}_{(S)_{+}}\left(F, V_{0}, w\right)=\operatorname{deg}_{(S)_{+}}\left(F, V_{0} \cap V_{1}, w\right)=\operatorname{deg}_{(S)_{+}}\left(F, V_{1}, w\right)
$$

and assertion $(b)$ also follows.

Therefore, if (6.1) holds, one can define $\widetilde{\operatorname{deg}}_{(S)_{+}}(F, U, w)$ as

$$
\widetilde{\operatorname{deg}}_{(S)_{+}}(F, U, w)=\operatorname{deg}_{(S)_{+}}(F, V, w) \text {, }
$$

where $V$ is any bounded and open subset of $X$ as in (a) of Proposition 6.10.

The next results are easy consequences of the properties of the degree in the previous setting.

Proposition 6.11. Assume that $U$ is bounded, $F: \bar{U} \rightarrow X^{\prime}$ is continuous and of $\operatorname{class}(S)_{+}$and $w \in X^{\prime} \backslash F(\partial U)$.

Then (6.1) holds and we have

$$
\widetilde{\operatorname{deg}}_{(S)_{+}}(F, U, w)=\operatorname{deg}_{(S)_{+}}(F, U, w),
$$

Proposition 6.12. If (6.1) holds, then

$$
\widetilde{\operatorname{deg}}_{(S)_{+}}(F, U, w)=\widetilde{\operatorname{deg}_{(S)_{+}}}(F-w, U, 0) .
$$

Theorem 6.13. If (6.1) holds, $u_{0} \in U$ and

$$
\left\langle F(u), u-u_{0}\right\rangle \geq\left\langle w, u-u_{0}\right\rangle \quad \text { for any } u \in U,
$$

then $\widetilde{\operatorname{deg}}_{(S)_{+}}(F, U, w)=1$.

Theorem 6.14. If

$$
\{u \in U: F(u)=w\}
$$

is empty, then $\widetilde{\operatorname{deg}_{(S)_{+}}}(F, U, w)=0$.

Theorem 6.15. If (6.1) holds and $U=U_{0} \cup U_{1}$, where $U_{0}, U_{1}$ are two disjoint open subsets of $X$, then

$$
\widetilde{\operatorname{deg}}_{(S)_{+}}(F, U, w)=\widetilde{\operatorname{deg}}_{(S)_{+}}\left(F, U_{0}, w\right)+\widetilde{\operatorname{deg}}_{(S)_{+}}\left(F, U_{1}, w\right) .
$$

Theorem 6.16. If (6.1) holds and $V$ is another open subset of $X$ with $V \subseteq U$ and $w \notin F(U \backslash V)$, then $\widetilde{\operatorname{deg}}_{(S)_{+}}(F, U, w)=\widetilde{\operatorname{deg}}_{(S)_{+}}(F, V, w)$.

Theorem 6.17. Assume that $H: U \times[0,1] \rightarrow X^{\prime}$ is continuous and locally of class $(S)_{+}$and that

$$
\left\{(u, t) \in U \times[0,1]: H_{t}(u)=w\right\}
$$

is compact (possibly empty).

Then $\widetilde{\operatorname{deg}}_{(S)_{+}}\left(H_{t}, U, w\right)$ is independent of $t \in[0,1]$. 
Theorem 6.18. If (6.1) holds with $w=0, U$ is symmetric with $0 \in U$ and $F$ is odd, then $\widetilde{\operatorname{deg}}_{(S)_{+}}(F, U, 0)$ is an odd integer.

\section{The COnstruction of the Degree}

Now consider again two Carathéodory functions

$$
a: \Omega \times \mathbb{R}^{N} \rightarrow \mathbb{R}^{N}, \quad b: \Omega \times\left(\mathbb{R} \times \mathbb{R}^{N}\right) \rightarrow \mathbb{R}
$$

satisfying $\left(a_{1}\right)-\left(a_{3}\right)$.

We first treat a particular case of (3.1), namely

$$
\begin{cases}-\operatorname{div}[a(x, \nabla u)]+b(x, u, \nabla u)=0 & \text { in } \Omega, \\ u=0 & \text { on } \partial \Omega .\end{cases}
$$

If we set

$$
\begin{array}{ll}
a_{\tau}(x, \xi)=a(x, \xi) & \text { if } 0 \leq \tau \leq 1, \\
b_{\tau}(x, s, \xi)=T_{1 / \tau}(b(x, s, \xi)) & \text { if } 0<\tau \leq 1, \\
b_{\tau}(x, s, \xi)=b(x, s, \xi) & \text { if } \tau=0,
\end{array}
$$

it is easily seen that $a_{\tau}, b_{\tau}$ satisfy $\left(u_{1}\right)-\left(u_{3}\right)$ with respect to $T=[0,1]$. Therefore we can consider the entropy solutions of

$$
\begin{cases}-\operatorname{div}[a(x, \nabla u)]+b_{\tau}(x, u, \nabla u)=0 & \text { in } \Omega, \\ u=0 & \text { on } \partial \Omega .\end{cases}
$$

Moreover, for every $\underline{\tau} \in] 0,1[$, we have

$$
\left|b_{\tau}(x, s, \xi)\right| \leq \frac{1}{\underline{\tau}} \quad \text { whenever } \underline{\tau} \leq \tau \leq 1 .
$$

Therefore, we can define a continuous map

$$
\left.\left.H: W_{0}^{1, p}(\Omega) \times\right] 0,1\right] \rightarrow W^{-1, p^{\prime}}(\Omega)
$$

by

$$
H_{\tau}(u)=-\operatorname{div}[a(x, \nabla u)]+b_{\tau}(x, u, \nabla u)
$$

and, according to $[10,24]$, this map is of class $(S)_{+}$(see also [1, Theorem 3.5]). Observe also that, if $U$ is an open subset of $\Phi_{0}^{1, p}(\Omega)$, then $U \cap W_{0}^{1, p}(\Omega)$ is an open subset of $W_{0}^{1, p}(\Omega)$.

Proposition 7.1. Let $U$ be a bounded and open subset of $\Phi_{0}^{1, p}(\Omega)$ such that (7.1) has no entropy solution $u \in \partial U$.

Then the following facts hold:

(a) there exists $\bar{\tau} \in] 0,1]$ such that (7.2) has no entropy solution with $0 \leq \tau \leq \bar{\tau}$ and $u \in \partial U$;

(b) if $\bar{\tau} \in] 0,1]$ is like in $(a)$, then for every $\underline{\tau} \in] 0, \bar{\tau}[$ the set

$$
\left\{(u, \tau) \in\left(U \cap W_{0}^{1, p}(\Omega)\right) \times[\underline{\tau}, \bar{\tau}]: H_{\tau}(u)=0\right\}
$$

is compact in $W_{0}^{1, p}(\Omega) \times[\underline{\tau}, \bar{\tau}]$ and the topological degree

$$
\widetilde{\operatorname{deg}}_{(S)_{+}}\left(H_{\tau}, U \cap W_{0}^{1, p}(\Omega), 0\right)
$$

is constant for $\tau \in] 0, \bar{\tau}]$. 
Proof. Since $\partial U$ is closed and bounded in $\Phi_{0}^{1, p}(\Omega)$, assertion $(a)$ follows from Corollary 5.2 .

On the other hand, for every $\underline{\tau} \in] 0, \bar{\tau}\left[\right.$, if $H_{\tau}(u)=0$ with $\tau \in[\underline{\tau}, \bar{\tau}]$ and $u \in$ $W_{0}^{1, p}(\Omega)$, it turns out that $u$ solves a problem of the form

$$
\begin{cases}-\operatorname{div}[a(x, \nabla u)]=z & \text { in } \Omega, \\ u=0 & \text { on } \partial \Omega,\end{cases}
$$

with $z \in L^{\infty}(\Omega)$ and $\|z\|_{\infty} \leq 1 / \underline{\tau}$. Since $\left\{(u, \tau) \mapsto H_{\tau}(u)\right\}$ is of class $(S)_{+}$, it easily follows that

$$
\left\{(u, \tau) \in W_{0}^{1, p}(\Omega) \times[\underline{\tau}, \bar{\tau}]: H_{\tau}(u)=0\right\}
$$

is compact in $W_{0}^{1, p}(\Omega) \times[\underline{\tau}, \bar{\tau}]$. Moreover, $H_{\tau}(u)=0$ implies that $(u, \tau)$ is an entropy solution of (7.2), so that there are no solutions of $H_{\tau}(u)=0$ with $u$ on the boundary of $U \cap W_{0}^{1, p}(\Omega)$ in $W_{0}^{1, p}(\Omega)$. Therefore

$$
\left\{(u, \tau) \in\left(U \cap W_{0}^{1, p}(\Omega)\right) \times[\underline{\tau}, \bar{\tau}]: H_{\tau}(u)=0\right\}
$$

also is compact in $W_{0}^{1, p}(\Omega) \times[\underline{\tau}, \bar{\tau}]$. By Theorem 6.17 we conclude that

$$
\widetilde{\operatorname{deg}}_{(S)_{+}}\left(H_{\tau}, U \cap W_{0}^{1, p}(\Omega), 0\right)
$$

is constant for $\tau \in[\underline{\tau}, \bar{\tau}]$, whenever $\underline{\tau} \in] 0, \bar{\tau}[$.

Definition 7.2. Let $U$ be a bounded and open subset of $\Phi_{0}^{1, p}(\Omega)$ such that (7.1) has no entropy solution $u \in \partial U$. We set

$$
\operatorname{deg}(-\operatorname{div}[a(x, \nabla u)]+b(x, u, \nabla u), U, 0)=\widetilde{\operatorname{deg}_{(S)_{+}}}\left(H_{\bar{\tau}}, U \cap W_{0}^{1, p}(\Omega), 0\right),
$$

where $\bar{\tau} \in] 0,1]$ is any number as in $(a)$ of the previous Proposition.

Theorem 7.3. Suppose that $\alpha_{2} \in L^{1}(\Omega) \cap W^{-1, p^{\prime}}(\Omega)$ in assumption $\left(a_{3}\right)$.

Then the following facts hold:

(a) we have

$$
\left\{\begin{array}{l}
b(x, u, \nabla u) v \in L^{1}(\Omega) \\
b(x, u, \nabla u) \in L^{1}(\Omega) \cap W^{-1, p^{\prime}}(\Omega)
\end{array} \quad \text { for any } u, v \in W_{0}^{1, p}(\Omega)\right.
$$

and the map

$$
\begin{array}{ccc}
W_{0}^{1, p}(\Omega) & \longrightarrow & W^{-1, p^{\prime}}(\Omega) \\
u & \mapsto & -\operatorname{div}[a(x, \nabla u)]+b(x, u, \nabla u)
\end{array}
$$

is continuous and of class $(S)_{+}$;

(b) every entropy solution of (7.1) belongs to $W_{0}^{1, p}(\Omega)$ and every $u \in W_{0}^{1, p}(\Omega)$ is an entropy solution of (7.1) if and only if

$$
-\operatorname{div}[a(x, \nabla u)]+b(x, u, \nabla u)=0 \quad \text { in } W^{-1, p^{\prime}}(\Omega) ;
$$

(c) if $U$ is a bounded and open subset of $\Phi_{0}^{1, p}(\Omega)$ such that (7.1) has no entropy solution $u \in \partial U$, then the set

$$
\{u \in U:-\operatorname{div}[a(x, \nabla u)]+b(x, u, \nabla u)=0\}
$$


is compact in $W_{0}^{1, p}(\Omega)$ and we have

$$
\begin{aligned}
\operatorname{deg}(-\operatorname{div}[a(x, \nabla u)] & +b(x, u, \nabla u), U, 0) \\
= & \operatorname{deg}_{(S)_{+}}(-\operatorname{div}[a(x, \nabla u)]+b(x, u, \nabla u), U \cap V, 0),
\end{aligned}
$$

whenever $V$ is a bounded and open subset of $W_{0}^{1, p}(\Omega)$ such that there are no solutions of (7.1) in $U \backslash V$.

Proof. First of all, it is easily seen that $\chi_{\left\{\alpha_{2} \geq k\right\}} \alpha_{2} \in L^{1}(\Omega) \cap W^{-1, p^{\prime}}(\Omega)$ for any $k>0$. We claim that

$$
\lim _{k \rightarrow+\infty} \chi_{\left\{\alpha_{2} \geq k\right\}} \alpha_{2}=0 \quad \text { strongly in } W^{-1, p^{\prime}}(\Omega) .
$$

Actually, let $v_{k} \in W_{0}^{1, p}(\Omega) \cap L^{\infty}(\Omega)$ be such that $\left\|\nabla v_{k}\right\|_{p} \leq 1$ and

$$
\left|\int_{\left\{\alpha_{2} \geq k\right\}} \alpha_{2} v_{k} d x\right| \geq \frac{1}{2}\left\|\chi_{\left\{\alpha_{2} \geq k\right\}} \alpha_{2}\right\|_{-1, p^{\prime}} .
$$

Up to a subsequence, $\left(v_{k}\right)$ is convergent to some $v$ weakly in $W_{0}^{1, p}(\Omega)$ and a.e. in $\Omega$, so that $\left(\left|v_{k}\right|\right)$ is convergent to $|v|$ weakly in $W_{0}^{1, p}(\Omega)$. Now $\left(\chi_{\left\{\alpha_{2} \geq k\right\}} \alpha_{2} v_{k}\right)$ is convergent to 0 a.e. in $\Omega$ and is dominated by $\left(\alpha_{2}\left|v_{k}\right|\right)$, which is convergent to $\alpha_{2}|v|$ a.e. in $\Omega$. According to [8], we also have $\alpha_{2}|v| \in L^{1}(\Omega)$ and

$$
\int_{\Omega} \alpha_{2}|v| d x=\left\langle\alpha_{2},|v|\right\rangle=\lim _{k}\left\langle\alpha_{2},\left|v_{k}\right|\right\rangle=\lim _{k} \int_{\Omega} \alpha_{2}\left|v_{k}\right| d x .
$$

By a variant of Lebesgue's theorem, we infer that

$$
\lim _{k} \int_{\left\{\alpha_{2} \geq k\right\}} \alpha_{2} v_{k} d x=0
$$

and (7.3) follows.

Now let $b_{\tau}$ be as before and let

$$
\begin{aligned}
& b_{\tau, 1}(x, s, \xi)=\min \left\{\max \left\{b_{\tau}(x, s, \xi),-\alpha_{2}(x)\right\}, \alpha_{2}(x)\right\} \\
& b_{\tau, 2}(x, s, \xi)=b_{\tau}(x, s, \xi)-b_{\tau, 1}(x, s, \xi) .
\end{aligned}
$$

It is easily seen that $b_{\tau, 1}, b_{\tau, 2}$ are Carathéodory functions satisfying

$$
\begin{aligned}
& b_{\tau}(x, s, \xi) \quad=b_{\tau, 1}(x, s, \xi)+b_{\tau, 2}(x, s, \xi), \\
& \left|b_{\tau, 1}(x, s, \xi)\right| \leq \alpha_{2}(x), \\
& \left|b_{\tau, 2}(x, s, \xi)\right| \leq \beta_{2}|s|^{r}+\beta_{2}|\xi|^{q} .
\end{aligned}
$$

Moreover, if $u \in \Phi_{0}^{1, p}(\Omega)$ and $v \in W_{0}^{1, p}(\Omega) \cap L^{\infty}(\Omega)$, we have

$$
\left|\int_{\Omega} b_{\tau, 1}(x, u, \nabla u) v d x\right| \leq \int_{\Omega} \alpha_{2}|v| d x \leq\left\|\alpha_{2}\right\|_{-1, p^{\prime}}\|[\nabla|v|]\|_{p}=\left\|\alpha_{2}\right\|_{-1, p^{\prime}}\|\nabla v\|_{p} .
$$

Therefore, it holds $b_{\tau, 1}(x, u, \nabla u) \in L^{1}(\Omega) \cap W^{-1, p^{\prime}}(\Omega)$ with

$$
\left\|b_{\tau, 1}(x, u, \nabla u)\right\|_{-1, p^{\prime}} \leq\left\|\alpha_{2}\right\|_{-1, p^{\prime}} .
$$

Moreover, again by [8], we have $\alpha_{2}|v| \in L^{1}(\Omega)$, hence $b_{\tau, 1}(x, u, \nabla u) v \in L^{1}(\Omega)$, for any $v \in W_{0}^{1, p}(\Omega)$.

If we set

$$
b_{\tau, 1}^{(k)}(x, s, \xi)=\chi_{\left\{\alpha_{2}<k\right\}}(x) b_{\tau, 1}(x, s, \xi),
$$


then $b_{\tau, 1}^{(k)}$ is a Carathéodory function and the same argument shows that

$$
\left\|b_{\tau, 1}^{(k)}(x, u, \nabla u)-b_{\tau, 1}(x, u, \nabla u)\right\|_{-1, p^{\prime}} \leq\left\|\chi_{\left\{\alpha_{2} \geq k\right\}} \alpha_{2}\right\|_{-1, p^{\prime}},
$$

so that

$$
\lim _{k}\left\|b_{\tau, 1}^{(k)}(x, u, \nabla u)-b_{\tau, 1}(x, u, \nabla u)\right\|_{-1, p^{\prime}}=0 \quad \text { uniformly for } u \in \Phi_{0}^{1, p}(\Omega)
$$

by (7.3). Since each map

$$
\begin{array}{ccc}
W_{0}^{1, p}(\Omega) \times[0,1] & \longrightarrow & W^{-1, p^{\prime}}(\Omega) \\
(u, \tau) & \mapsto & b_{\tau, 1}^{(k)}(x, u, \nabla u)
\end{array}
$$

is completely continuous, as $\left|b_{\tau, 1}^{(k)}(x, s, \xi)\right| \leq k$, it follows that

$$
\begin{array}{cl}
W_{0}^{1, p}(\Omega) \times[0,1] & \longrightarrow W^{-1, p^{\prime}}(\Omega) \\
(u, \tau) & \mapsto b_{\tau, 1}(x, u, \nabla u)
\end{array}
$$

is completely continuous, too.

On the other hand, it is standard that

$$
\left\{\begin{array}{l}
b_{\tau, 2}(x, u, \nabla u) v \in L^{1}(\Omega) \\
b_{\tau, 2}(x, u, \nabla u) \in L^{1}(\Omega) \cap W^{-1, p^{\prime}}(\Omega)
\end{array} \quad \text { for any } u, v \in W_{0}^{1, p}(\Omega)\right.
$$

and that the map

$$
\begin{array}{ccc}
W_{0}^{1, p}(\Omega) \times[0,1] & \longrightarrow & W^{-1, p^{\prime}}(\Omega) \\
(u, \tau) & \mapsto & -\operatorname{div}\left[a(x, \nabla u)+b_{\tau, 2}(x, u, \nabla u)\right.
\end{array}
$$

is continuos and of class $(S)_{+}$(see e.g. $\left.[10,24]\right)$. Then

$$
\begin{array}{ccc}
W_{0}^{1, p}(\Omega) \times[0,1] & \longrightarrow & W^{-1, p^{\prime}}(\Omega) \\
(u, \tau) & \mapsto & -\operatorname{div}\left[a(x, \nabla u)+b_{\tau}(x, u, \nabla u)\right.
\end{array}
$$

is continuos and of class $(S)_{+}$, too, and assertion $(a)$ follows.

For every $u \in \Phi_{0}^{1, p}(\Omega)$ and $\tau \in[0,1]$, there exists $w_{u, \tau} \in L^{p^{\prime}}\left(\Omega ; \mathbb{R}^{N}\right)$ such that

$$
b_{\tau, 1}(x, u, \nabla u)=\operatorname{div} w_{u, \tau}
$$

and $\left\|w_{u, \tau}\right\|_{p^{\prime}}$ is bounded by a constant independent of $u$ and $\tau$. Therefore, each entropy solution of (7.2) is also an entropy solution of

$$
\begin{cases}-\operatorname{div}[\tilde{a}(x, \nabla u)]+b_{\tau, 2}(x, u, \nabla u)=0 & \text { in } \Omega, \\ u=0 & \text { on } \partial \Omega,\end{cases}
$$

with

$$
\tilde{a}(x, \xi)=a(x, \xi)-w_{u, \tau}(x),
$$

and $\tilde{a}$ also satisfies $\left(a_{1}\right)$ and $\left(a_{2}\right)$, possibly with different $\alpha_{0}, \alpha_{1}, \beta_{1}$ and $\nu$, where $\left\|\alpha_{0}\right\|_{1},\left\|\alpha_{1}\right\|_{p^{\prime}}, \beta_{1}$ and $1 / \nu$ are bounded by a constant independent of $u$ and $\tau$. By Theorem 2.10 and a standard bootstrap argument, we infer that $u \in W_{0}^{1, p}(\Omega)$. Moreover, if $B$ is a bounded subset of $\Phi_{0}^{1, p}(\Omega)$, we have that

$$
\{(u, \tau) \in B \times[0,1]:(u, \tau) \text { is an entropy solution of }(7.2)\}
$$

is bounded in $W_{0}^{1, p}(\Omega) \times[0,1]$. 
As in [3], any $u \in W_{0}^{1, p}(\Omega)$ is an entropy solution of (7.1) if and only if

$$
-\operatorname{div}[a(x, \nabla u)]+b(x, u, \nabla u)=0 \quad \text { in } W^{-1, p^{\prime}}(\Omega)
$$

and assertion (b) follows.

To prove assertion $(c)$, observe that, since $U$ is bounded in $\Phi_{0}^{1, p}(\Omega)$, we have that the set

$$
\{(u, \tau) \in U \times[0,1]:(u, \tau) \text { is an entropy solution of }(7.2)\}
$$

is bounded in $W_{0}^{1, p}(\Omega) \times[0,1]$.

According to Proposition 7.1 , let now $\bar{\tau} \in] 0,1]$ be such that $(7.2)$ has no entropy solution with $0 \leq \tau \leq \bar{\tau}$ and $u \in \partial U$. Since the map

$$
\left\{(u, \tau) \mapsto-\operatorname{div}\left[a(x, \nabla u)+b_{\tau}(x, u, \nabla u)\right\}\right.
$$

is continuous and of class $(S)_{+}$, the set

$$
\{(u, \tau) \in U \times[0, \bar{\tau}]:(u, \tau) \text { is an entropy solution of }(7.2)\}
$$

is even compact in $W_{0}^{1, p}(\Omega) \times[0, \bar{\tau}]$. Let $V$ be a bounded and open subset of $W_{0}^{1, p}(\Omega)$ such that there are no solutions of (7.1) in $U \backslash V$ and let $W$ be a bounded and open subset of $W_{0}^{1, p}(\Omega)$ such that $\bar{W} \subseteq U$ and there are no solutions of (7.2) with $0 \leq t \leq \bar{\tau}$ and $u \in U \backslash W$. Combining Definition 7.2 with Theorems 6.7 and 6.8, we conclude that

$$
\begin{aligned}
& \operatorname{deg}(-\operatorname{div}[a(x, \nabla u)]+b(x, u, \nabla u), U, 0) \\
&=\widetilde{\operatorname{deg}_{(S)_{+}}\left(H_{\bar{\tau}}, U \cap W_{0}^{1, p}(\Omega), 0\right)}=\operatorname{deg}_{(S)_{+}}\left(H_{\bar{\tau}}, W, 0\right) \\
&=\operatorname{deg}_{(S)_{+}}(-\operatorname{div}[a(x, \nabla u)]+b(x, u, \nabla u), W, 0) \\
&=\operatorname{deg}_{(S)_{+}}(-\operatorname{div}[a(x, \nabla u)]+b(x, u, \nabla u), W \cap V, 0) \\
&=\operatorname{deg}_{(S)_{+}}(-\operatorname{div}[a(x, \nabla u)]+b(x, u, \nabla u), U \cap V, 0)
\end{aligned}
$$

and assertion $(c)$ follows.

Theorem 7.4. Let $U$ be a bounded and open subset of $\Phi_{0}^{1, p}(\Omega)$ such that the equation (7.1) has no entropy solution $u \in \bar{U}$.

Then

$$
\operatorname{deg}(-\operatorname{div}[a(x, \nabla u)]+b(x, u, \nabla u), U, 0)=0 .
$$

Proof. Since $\bar{U}$ is closed and bounded in $\Phi_{0}^{1, p}(\Omega)$, by Corollary 5.2 there exists $\bar{\tau} \in] 0,1]$ such that (7.2) has no entropy solution with $0 \leq \tau \leq \bar{\tau}$ and $u \in \bar{U}$. In particular, the equation $H_{\bar{\tau}}(u)=0$ has no solution $u \in U \cap W_{0}^{1, p}(\Omega)$. By Theorem 6.14 it follows

$$
\operatorname{deg}(-\operatorname{div}[a(x, \nabla u)]+b(x, u, \nabla u), U, 0)=\widetilde{\operatorname{deg}_{(S)_{+}}}\left(H_{\bar{\tau}}, U \cap W_{0}^{1, p}(\Omega), 0\right)=0 .
$$

In a similar way, Theorem 3.8 and the next two results can be proved taking advantage of Theorems 6.18, 6.15, 6.16 and Corollary 5.2.

Theorem 7.5. Let $U$ be a bounded and open subset of $\Phi_{0}^{1, p}(\Omega)$ such that (7.1) has no entropy solution $u \in \partial U$. Assume that $U=U_{1} \cup U_{2}$, where $U_{1}, U_{2}$ are two disjoint open subsets of $\Phi_{0}^{1, p}(\Omega)$. 
Then

$$
\begin{aligned}
\operatorname{deg}(-\operatorname{div}[a(x, \nabla u)] & +b(x, u, \nabla u), U, 0) \\
= & \operatorname{deg}\left(-\operatorname{div}[a(x, \nabla u)]+b(x, u, \nabla u), U_{1}, 0\right) \\
& +\operatorname{deg}\left(-\operatorname{div}[a(x, \nabla u)]+b(x, u, \nabla u), U_{2}, 0\right) .
\end{aligned}
$$

Theorem 7.6. Let $V \subseteq U$ be two bounded and open subsets of $\Phi_{0}^{1, p}(\Omega)$ such that (7.1) has no entropy solution $u \in \bar{U} \backslash V$.

Then

$$
\begin{aligned}
\operatorname{deg}(-\operatorname{div}[a(x, \nabla u)]+b(x, u, \nabla u), U, 0) & \\
& =\operatorname{deg}(-\operatorname{div}[a(x, \nabla u)]+b(x, u, \nabla u), V, 0) .
\end{aligned}
$$

Let us see more in detail the homotopy invariance.

Theorem 7.7. Let

$$
a: \Omega \times\left(\mathbb{R}^{N} \times[0,1]\right) \rightarrow \mathbb{R}^{N}, \quad b: \Omega \times\left(\mathbb{R} \times \mathbb{R}^{N} \times[0,1]\right) \rightarrow \mathbb{R}
$$

be two Carathéodory functions satisfying $\left(u_{1}\right)-\left(u_{3}\right)$.

Then the following facts hold:

(a) for every bounded and closed subset $C$ of $\Phi_{0}^{1, p}(\Omega)$, the set of $t$ 's in $[0,1]$ such that

$$
\begin{cases}-\operatorname{div}\left[a_{t}(x, \nabla u)\right]+b_{t}(x, u, \nabla u)=0 & \text { in } \Omega, \\ u=0 & \text { on } \partial \Omega\end{cases}
$$

admits an entropy solution $u \in C$ is closed in $[0,1]$;

(b) for every bounded and open subset $U$ of $\Phi_{0}^{1, p}(\Omega)$, if (7.4) has no entropy solution with $t \in[0,1]$ and $u \in \partial U$, then

$$
\operatorname{deg}\left(-\operatorname{div}\left[a_{t}(x, \nabla u)\right]+b_{t}(x, u, \nabla u), U, 0\right)
$$

is independent of $t \in[0,1]$.

Proof. Assertion ( $a$ ) is a particular case of Corollary 5.2. To prove (b), consider

$$
\begin{array}{ll}
a_{t, \tau}(x, \xi)=a_{t}(x, \xi) & \text { if } 0 \leq \tau \leq 1, \\
b_{t, \tau}(x, s, \xi)=T_{1 / \tau}\left(b_{t}(x, s, \xi)\right) & \text { if } 0<\tau \leq 1, \\
b_{t, \tau}(x, s, \xi)=b_{t}(x, s, \xi) & \text { if } \tau=0,
\end{array}
$$

for $(t, \tau) \in T=[0,1] \times[0,1]$. It is easily seen that $a_{t, \tau}$ and $b_{t, \tau}$ satisfy $\left(u_{1}\right)-\left(u_{3}\right)$.

Define also

$$
H_{t, \tau}(u)=-\operatorname{div}\left[a_{t, \tau}(x, \nabla u)\right]+b_{t, \tau}(x, u, \nabla u),
$$

for $0 \leq t \leq 1,0<\tau \leq 1$ and $u \in W_{0}^{1, p}(\Omega)$.

Since $[0,1] \times\{0\}$ is compact, by Corollary 5.2 there exists $\bar{\tau} \in] 0,1]$ such that

$$
\begin{cases}-\operatorname{div}\left[a_{t, \tau}(x, \nabla u)\right]+b_{t, \tau}(x, u, \nabla u)=0 & \text { in } \Omega, \\ u=0 & \text { on } \partial \Omega,\end{cases}
$$

has no entropy solution with $(t, \tau) \in[0,1] \times[0, \bar{\tau}]$ and $u \in \partial U$.

For any $t \in[0,1]$, it follows

$$
\operatorname{deg}\left(-\operatorname{div}\left[a_{t}(x, \nabla u)\right]+b_{t}(x, u, \nabla u), U, 0\right)=\widetilde{\operatorname{deg}}_{(S)_{+}}\left(H_{t, \bar{\tau}}, U \cap W_{0}^{1, p}(\Omega), 0\right) .
$$


On the other hand, as before, the map $\left\{(u, t) \mapsto H_{t, \bar{\tau}}(u)\right\}$ is continuous and of class $(S)_{+}$and the set

$$
\left\{(u, t) \in\left(U \cap W_{0}^{1, p}(\Omega)\right) \times[0,1]: H_{t, \bar{\tau}}(u)=0\right\}
$$

is compact in $W_{0}^{1, p}(\Omega) \times[0,1]$. By Theorem 6.17 we conclude that

$$
\widetilde{\operatorname{deg}}_{(S)_{+}}\left(H_{t, \bar{\tau}}, U \cap W_{0}^{1, p}(\Omega), 0\right)
$$

is independent of $t$.

Corollary 7.8. Let $U$ be a bounded and open subset of $\Phi_{0}^{1, p}(\Omega)$ such that (7.1) has no entropy solution $u \in \partial U$. Let also

$$
\hat{a}: \Omega \times \mathbb{R}^{N} \rightarrow \mathbb{R}^{N}, \quad \hat{b}: \Omega \times\left(\mathbb{R} \times \mathbb{R}^{N}\right) \rightarrow \mathbb{R}
$$

be two Carathéodory functions satisfying $\left(a_{1}\right)-\left(a_{3}\right)$ and such that

$$
\begin{array}{r}
\int_{\Omega}\{a(x, \nabla z) \cdot \nabla v+b(x, z, \nabla z) v\} d x=\int_{\Omega}\{\hat{a}(x, \nabla z) \cdot \nabla v+\hat{b}(x, z, \nabla z) v\} d x \\
\forall z, v \in W_{0}^{1, p}(\Omega) \cap L^{\infty}(\Omega) .
\end{array}
$$

Then

$$
\begin{aligned}
\operatorname{deg}(-\operatorname{div}[a(x, \nabla u)]+b(x, u, \nabla u), U, 0) & \\
& =\operatorname{deg}(-\operatorname{div}[\hat{a}(x, \nabla u)]+\hat{b}(x, u, \nabla u), U, 0) .
\end{aligned}
$$

Proof. Taking into account Remark 3.1, it is enough to apply Theorem 7.7 to

$$
a_{t}(x, \xi)=(1-t) a(x, \xi)+t \hat{a}(x, \xi), \quad b_{t}(x, s, \xi)=(1-t) b(x, s, \xi)+t \hat{b}(x, s, \xi) .
$$

Now we treat (3.1) in the general case. According to [7], any $\mu \in \mathcal{M}_{b}^{p}(\Omega)$ satisfies

$$
\int_{\Omega} v d \mu=\int_{\Omega} v w_{0} d x+\int_{\Omega}(\nabla v) \cdot w_{1} d x \quad \forall v \in W_{0}^{1, p}(\Omega) \cap L^{\infty}(\Omega),
$$

for some $w_{0} \in L^{1}(\Omega)$ and $w_{1} \in L^{p^{\prime}}\left(\Omega ; \mathbb{R}^{N}\right)$. On the other hand, if $w_{0} \in L^{1}(\Omega)$ and $w_{1} \in L^{p^{\prime}}\left(\Omega ; \mathbb{R}^{N}\right)$, it is easily seen that the functions

$$
\tilde{a}(x, \xi)=a(x, \xi)-w_{1}(x), \quad \tilde{b}(x, s, \xi)=b(x, s, \xi)-w_{0}(x)
$$

still satisfy $\left(a_{1}\right)-\left(a_{3}\right)$.

Proposition 7.9. Let $\mu \in \mathcal{M}_{b}^{p}(\Omega)$ and let $U$ be a bounded and open subset of $\Phi_{0}^{1, p}(\Omega)$ such that (3.1) has no entropy solution $u \in \partial U$. Let also $w_{0}, \hat{w}_{0} \in L^{1}(\Omega)$ and $w_{1}, \hat{w}_{1} \in L^{p^{\prime}}\left(\Omega ; \mathbb{R}^{N}\right)$ be such that

$$
\begin{aligned}
& \int_{\Omega} v d \mu=\int_{\Omega} v w_{0} d x+\int_{\Omega}(\nabla v) \cdot w_{1} d x=\int_{\Omega} v \hat{w}_{0} d x+ \int_{\Omega}(\nabla v) \cdot \hat{w}_{1} d x \\
& \forall v \in W_{0}^{1, p}(\Omega) \cap L^{\infty}(\Omega) .
\end{aligned}
$$

Then

$$
\begin{aligned}
\operatorname{deg}\left(-\operatorname{div}\left[a(x, \nabla u)-w_{1}\right]\right. & \left.+b(x, u, \nabla u)-w_{0}, U, 0\right) \\
& =\operatorname{deg}\left(-\operatorname{div}\left[a(x, \nabla u)-\hat{w}_{1}\right]+b(x, u, \nabla u)-\hat{w}_{0}, U, 0\right) .
\end{aligned}
$$

Proof. It is a consequence of Corollary 7.8. 
Definition 7.10. Let $\mu \in \mathcal{M}_{b}^{p}(\Omega)$ and let $U$ be a bounded and open subset of $\Phi_{0}^{1, p}(\Omega)$ such that (3.1) has no entropy solution $u \in \partial U$.

We set

$$
\begin{aligned}
\operatorname{deg}(-\operatorname{div}[a(x, \nabla u)]+b(x, u, \nabla u), U, \mu) & \\
& =\operatorname{deg}\left(-\operatorname{div}\left[a(x, \nabla u)-w_{1}\right]+b(x, u, \nabla u)-w_{0}, U, 0\right),
\end{aligned}
$$

where $w_{0} \in L^{1}(\Omega)$ and $w_{1} \in L^{p^{\prime}}\left(\Omega ; \mathbb{R}^{N}\right)$ satisfy

$$
\int_{\Omega} v d \mu=\int_{\Omega} v w_{0} d x+\int_{\Omega}(\nabla v) \cdot w_{1} d x \quad \forall v \in W_{0}^{1, p}(\Omega) \cap L^{\infty}(\Omega) .
$$

Theorems 3.2, 3.4, 3.5, 3.6 and 3.7 can be easily reduced to Theorems 7.3, 7.4, 7.5, 7.6 and 7.7, respectively. Let us see in detail the normalization property.

Proof of Theorem 3.3.

If we set

$$
a_{t}(x, \xi)=a(x, \xi)-(1-t) a(x, 0), \quad b_{t}(x, s, \xi)=0, \quad \mu_{0}=0, \quad \mu_{1}=\mu,
$$

it is easily seen that $a_{t}, b_{t}$ satisfy $\left(u_{1}\right)-\left(u_{3}\right)$, possibly with $\nu, \alpha_{0}, \alpha_{1}$ and $\beta_{1}$ replaced by some $\hat{\nu}, \hat{\alpha}_{0}, \hat{\alpha}_{1}$ and $\hat{\beta}_{1}$. If we set

$$
V=U \cup\left\{v \in \Phi_{0}^{1, p}(\Omega): \hat{\nu}\left\|\nabla\left[\varphi_{p}(v)\right]\right\|_{p}<\|\psi\|_{\infty}|\mu|(\Omega)+\left\|\hat{\alpha}_{0}\right\|_{1}+1\right\},
$$

by (2.5) there is no entropy solution of (3.2) with $t \in[0,1]$ and $u \in \partial V$. By Theorem 3.7, it follows

$$
\operatorname{deg}(-\operatorname{div}[a(x, \nabla u)], V, \mu)=\operatorname{deg}(-\operatorname{div}[a(x, \nabla u)-a(x, 0)], V, 0)
$$

and we also have

$$
\operatorname{deg}(-\operatorname{div}[a(x, \nabla u)], V, \mu)=\operatorname{deg}(-\operatorname{div}[a(x, \nabla u)], U, \mu)
$$

by Theorem 3.6.

On the other hand, if $W$ is any bounded and open neighborhood of 0 in $W_{0}^{1, p}(\Omega)$, by Theorem 3.2 it holds

$$
\begin{aligned}
& \operatorname{deg}(-\operatorname{div}[a(x, \nabla u)-a(x, 0)], V, 0) \\
& \quad=\operatorname{deg}_{(S)_{+}}(-\operatorname{div}[a(x, \nabla u)-a(x, 0)], V \cap W, 0)
\end{aligned}
$$

and, finally,

$$
\operatorname{deg}_{(S)_{+}}(-\operatorname{div}[a(x, \nabla u)-a(x, 0)], V \cap W, 0)=1
$$

by Theorem 6.4 with $u_{0}=0$.

\section{REFERENCES}

[1] S. Almi And M. Degiovanni, On degree theory for quasilinear elliptic equations with natural growth conditions, in Recent Trends in Nonlinear Partial Differential Equations II: Stationary Problems, J.B. Serrin, E.L. Mitidieri and V.D. Rădulescu eds., 1-20, Contemp. Math., 595, Amer. Math. Soc., Providence, R.I., 2013.

[2] H. Amann And P. Quittner, Elliptic boundary value problems involving measures: existence, regularity, and multiplicity, Adv. Differential Equations 3 (1998), no. 6, 753-813.

[3] P. Bénilan, L. Boccardo, T. Gallouët, R. Gariepy, M. Pierre and J. L. Vázquez, An $L^{1}$-theory of existence and uniqueness of solutions of nonlinear elliptic equations, Ann. Scuola Norm. Sup. Pisa Cl. Sci. (4) 22 (1995), no. 2, 241-273.

[4] L. Boccardo, P. Drábek, D. Giachetti and M. KuČera, Generalization of Fredholm alternative for nonlinear differential operators, Nonlinear Anal. 10 (1986), no. 10, 1083-1103. 
[5] L. Boccardo and T. Gallouët, Nonlinear elliptic and parabolic equations involving measure data, J. Funct. Anal. 87 (1989), no. 1, 149-169.

[6] L. Boccardo And T. Galloü̈t, Nonlinear elliptic equations with right hand side measures, Comm. Partial Differential Equations 17 (1992), no. 3-4, 641-655.

[7] L. Boccardo, T. Gallouët and L. Orsina, Existence and uniqueness of entropy solutions for nonlinear elliptic equations with measure data, Ann. Inst. H. Poincaré Anal. Non Linéaire 13 (1996), no. 5, 539-551.

[8] H. Brezis and F. E. Browder, Sur une propriété des espaces de Sobolev, C. R. Acad. Sci. Paris Sér. A-B 287 (1978), no. 3, A113-A115.

[9] H. Brezis, M. Marcus And A. C. Ponce, Nonlinear elliptic equations with measures revisited, in Mathematical aspects of nonlinear dispersive equations, J. Bourgain, C.E. Kenig and S. Klainerman eds., 55-109, Ann. of Math. Stud., 163, Princeton Univ. Press, Princeton, NJ, 2007.

[10] F.E. Browder, Fixed point theory and nonlinear problems, Bull. Amer. Math. Soc. (N.S.) 9 (1983), no. 1, 1-39.

[11] G. Dal Maso and F. Murat, Almost everywhere convergence of gradients of solutions to nonlinear elliptic systems, Nonlinear Anal. 31 (1998), no. 3-4, 405-412.

[12] G. Dal Maso, F. Murat, L. Orsina and A.Prignet, Renormalized solutions of elliptic equations with general measure data, Ann. Scuola Norm. Sup. Pisa Cl. Sci. (4) 28 (1999), no. 4, 741-808.

[13] M. Degiovanni and M. Scaglia, A variational approach to semilinear elliptic equations with measure data, Discrete Contin. Dyn. Syst. 31 (2011), no. 4, 1233-1248.

[14] J. Dugundu And A. Granas, "Fixed point theory", Springer Monographs in Mathematics, Springer-Verlag, New York, 2003.

[15] A. Ferrero And C. SACCON, Existence and multiplicity results for semilinear equations with measure data, Topol. Methods Nonlinear Anal. 28 (2006), no. 2, 285-318.

[16] A. Ferrero and C. SACCON, Existence and multiplicity results for semilinear elliptic equations with measure data and jumping nonlinearities, Topol. Methods Nonlinear Anal. 30 (2007), no. 1, 37-65.

[17] A. Ferrero And C. SACCON, Multiplicity results for a class of asymptotically linear elliptic problems with resonance and applications to problems with measure data, Adv. Nonlinear Stud. 10 (2010), no. 2, 433-479.

[18] L. Greco, T. Iwaniec and C. Sbordone, Inverting the p-harmonic operator, Manuscripta Math. 92 (1997), no. 2, 249-258.

[19] T. KilpeläInen and X. XU, On the uniqueness problem for quasilinear elliptic equations involving measures, Rev. Mat. Iberoamericana 12 (1996), no. 2, 461-475.

[20] A. Malusa And A, Prignet, Stability of renormalized solutions of elliptic equations with measure data, Atti Semin. Mat. Fis. Univ. Modena Reggio Emilia 52 (2004), no. 1, 151-168.

[21] M. Marcus And L. VÉRon, "Nonlinear second order elliptic equations involving measures", De Gruyter Series in Nonlinear Analysis and Applications, 21, De Gruyter, Berlin, 2014.

[22] D. O'Regan, Y.J. Cho And Y.-Q. Chen, "Topological degree theory and applications", Series in Mathematical Analysis and Applications, 10, Chapman \& Hall/CRC, Boca Raton, FL, 2006.

[23] L. Orsina, Solvability of linear and semilinear eigenvalue problems with $L^{1}$ data, Rend. Sem. Mat. Univ. Padova 90 (1993), 207-238.

[24] I.V. SkRYPNIK, "Methods for analysis of nonlinear elliptic boundary value problems", Translations of Mathematical Monographs, 139, American Mathematical Society, Providence, RI, 1994.

[25] P. TAKÁč, Variational methods and linearization tools towards the spectral analysis of the $p$-Laplacian, especially for the Fredholm alternative, in Proceedings of the 2007 Conference on Variational and Topological Methods: Theory, Applications, Numerical Simulations, and Open Problems, M. Chhetri, P. Girg and J.M. Neuberger eds., 67-105, Electron. J. Differ. Equ. Conf., 18, Southwest Texas State Univ., San Marcos, TX, 2010.

[26] L. VÉron, Elliptic equations involving measures, in Stationary partial differential equations. Vol. I, M. Chipot and P. Quittner eds., 593-712, Handb. Differ. Equ., North-Holland, Amsterdam, 2004. 
Dipartimento di Matematica "F. Casorati", Università di Pavia, Via Ferrata 5, 27100 PAVIA, ITALY

E-mail address: michele.colturato01@universitadipavia.it

Dipartimento di Matematica e Fisica, Università Cattolica del Sacro Cuore, Via dei Musei 41, 25121 Brescia, Italy

E-mail address: marco.degiovanni@unicatt.it 